\title{
Asymptotic behavior and regularity properties of strongly nonlinear parabolic equations
}

\author{
Maria Michaela Porzio ${ }^{1}$
}

Received: 27 April 2018 / Accepted: 23 March 2019 / Published online: 1 April 2019

(c) Fondazione Annali di Matematica Pura ed Applicata and Springer-Verlag GmbH Germany, part of Springer Nature 2019

\begin{abstract}
In this paper, we study a class of nonlinear parabolic problems including the $p$-Laplacian equation. The initial datum and the forcing term are allowed to be summable functions or Radon measures. We prove that these equations have surprising regularizing properties. Moreover, we study the behavior in time of these solutions proving that decay estimates hold true also for nonzero reaction terms. Finally, we study the autonomous case.
\end{abstract}

Keywords Decay estimates - Asymptotic behavior · Regularity of solutions $\cdot p$-Laplacian equation $\cdot$ Nonlinear degenerate parabolic equations $\cdot$ Smoothing effect

Mathematics Subject Classification $35 \mathrm{~K} 10 \cdot 35 \mathrm{~K} 55 \cdot 35 \mathrm{~K} 65 \cdot 35 \mathrm{~K} 58$

\section{Introduction}

Let us consider the following nonlinear parabolic problem

$$
\begin{cases}u_{t}-\operatorname{div}(a(x, t, \nabla u))=f(x, t) & \text { in } \Omega_{T}, \\ u=0 & \text { on } \Gamma, \\ u(x, 0)=u_{0}(x) & \text { on } \Omega,\end{cases}
$$

where $\Omega_{T}=\Omega \times(0, T), \Omega$ is an open bounded set of $\mathbb{R}^{N}, N>2, T>0$ and $\Gamma=\partial \Omega \times(0, T)$, with $\partial \Omega$ regular.

Here the function $a(x, t, \xi): \Omega \times(0, T) \times \mathbb{R}^{N} \rightarrow \mathbb{R}^{N}$ is a Caratheodory function ${ }^{1}$ satisfying, for a.e. $(x, t) \in \Omega_{T}$ and for every $\xi$ and $\eta \in \mathbb{R}^{N}$ the following structure conditions

$$
\begin{aligned}
a(x, t, 0) & =0, \\
{[a(x, t, \xi)-a(x, t, \eta)][\xi-\eta] } & \geq \alpha|\xi-\eta|^{p}, \quad \alpha>0, \quad 2<p<N,
\end{aligned}
$$

1 i.e., it is continuous with respect to $\xi$ for almost every $(x, t) \in \Omega_{T}$, and measurable with respect to $(x, t)$ for every $\xi \in \mathbb{R}^{N}$.

$凶 \quad$ Maria Michaela Porzio

porzio@mat.uniroma1.it

1 Dipartimento di Matematica "Guido Castelnuovo", Sapienza Universitá di Roma, Piazzale A. Moro 2, 00185 Rome, Italy 
and

$$
|a(x, t, \xi)| \leq \beta\left[|\xi|^{p-1}+h(x, t)\right], \quad \beta>0, \quad h \in L^{p^{\prime}}\left(\Omega_{T}\right) \quad \frac{1}{p}+\frac{1}{p^{\prime}}=1
$$

Notice that assumptions (1.2) and (1.3) are equivalent to require that (1.3) and the following coercivity condition hold true

$$
a(x, t, \xi) \xi \geq \alpha|\xi|^{p}
$$

since (1.5) implies (1.2).

We recall that under the previous structural assumptions, also in presence of only summable data $f \in L^{1}\left(\Omega_{T}\right)$ and $u_{0} \in L^{1}(\Omega)$, there exists at least a solution $u$ of (1.1) (see [7] and Definition 2.1). Generally, these solutions are not unique. To guarantee the uniqueness further requirements are needed even when the forcing term $f$ is identically zero (see $[1,3-5,11,21,28]$ and the references therein).

The model we have in mind is the p-Laplacian equation

$$
\begin{cases}u_{t}-\Delta_{p} u=f(x, t) & \text { in } \Omega_{T}, \\ u=0 & \text { on } \Gamma, \\ u(x, 0)=u_{0}(x) & \text { on } \Omega,\end{cases}
$$

which is widely studied since it appears in a lot of applicative problems modeling diffusive problems arising in various different contexts like, for example, fluid dynamics (non-Newtonian fluids), biology (evolution of biological species and populations), hydrology, glaciology etc. (see [29] and the references therein).

It is well known that in the absence of the forcing term f, i.e., when (1.6) becomes

$$
\begin{cases}u_{t}=\Delta_{p} u & \text { in } \Omega_{T}, \\ u=0 & \text { on } \Gamma, \\ u(x, 0)=u_{0}(x) & \text { on } \Omega,\end{cases}
$$

a very strong regularization property appears: there exists a solution $u$ of (1.7) which becomes "immediately bounded" also if the initial datum $u_{0}$ is only a summable function (see [15] if $N=1,[9,13,14,20,29,30]$ and the references therein). Moreover, this solution satisfies the following decay estimate

$$
\|u(t)\|_{L^{\infty}(\Omega)} \leq c . \frac{\left\|u_{0}\right\|_{L^{1}(\Omega)}^{\frac{p}{N(p-2)+p}}}{t^{\frac{N}{N(p-2)+p}}}
$$

(see [20,29]).

Indeed, also the following bound holds true

$$
\|u(t)\|_{L^{\infty}(\Omega)} \leq \frac{c}{t^{\frac{1}{p-2}}}
$$

where $c$ depends only on $N, p, \alpha$ and $|\Omega|$ (see [20]). This last estimate is noticeable different from (1.8) because it does not depend on the initial datum $u_{0}$. Bounds like (1.9) (i.e., independent of $u_{0}$ ) are often referred in the literature as "universal estimates". Notice that the value $\frac{1}{p-2}$ is larger than the decay exponent that appears in (1.8) and consequently this last inequality is a better estimate for large values of $t$.

Finally, all the previous results remain true for problem (1.7) replaced by

$$
\begin{cases}u_{t}=\operatorname{div}(a(x, t, \nabla u)) & \text { in } \Omega_{T}, \\ u=0 & \text { on } \Gamma, \\ u(x, 0)=u_{0}(x) & \text { on } \Omega,\end{cases}
$$


when (1.2)-(1.4) are retained and also estimates (1.8)-(1.9) still hold true in this more general setting (see $[10,20])$.

In addition, it is also unique the solution that has this regularizing property (see [21]) and it is also possible to show that if the summability of the initial datum increases, for example if $u_{0}$ belongs to $L^{r_{0}}(\Omega)$ with $r_{0}>1$, then this higher summability influences the decay estimates (1.8) that becomes

$$
\|u(t)\|_{L^{\infty}(\Omega)} \leq c \frac{\left\|u_{0}\right\|_{L^{r_{0}(\Omega)}}^{\frac{p r_{0}}{N(p-2)+p r_{0}}}}{t^{\frac{N r_{0}}{N(p-2)+p r_{0}}}}
$$

(see [20]).

We point out that, although this equation is widely studied, it seems an open problem to understand what happens to these decay estimates and to this strong $L^{\infty}$-regularization property when $f$ is not identically zero and the initial datum $u_{0}$ belongs to $L^{1}(\Omega)$.

Hence, this "open case" is the contempt of this paper.

We prove here that if $\mathrm{f}$ is a function satisfying

$$
f \in L^{m}\left(0, T ; L^{s}(\Omega)\right) \quad s \geq 1 \quad m \geq 1
$$

and

$$
u_{0} \in L^{1}(\Omega)
$$

then there exists a solution of (1.1) which immediately increases its regularity reaching the same summability properties of the solution $u^{0}$ (obtained by approximation) of the following problem

$$
\begin{cases}\left(u^{0}\right)_{t}-\operatorname{div}\left(a\left(x, t, \nabla u^{0}\right)\right)=f(x, t) & \text { in } \Omega_{T}, \\ u^{0}=0 & \text { on } \Gamma, \\ u^{0}(x, 0)=0 & \text { on } \Omega .\end{cases}
$$

Hence, for every $\varepsilon \in(0, T)$ we have

$$
u^{0} \in L^{\delta}\left(\varepsilon, T ; L^{v}(\Omega)\right) \quad \Longrightarrow \quad u \in L^{\delta}\left(\varepsilon, T ; L^{\nu}(\Omega)\right) .
$$

In particular, when $f \equiv 0$ being $u^{0} \in L^{\infty}\left(\Omega_{T}\right)$ (indeed in this case $u^{0} \equiv 0$ ) we obtain the $L^{\infty}$-regularization property recalled above for null data f. Notice that $u^{0}$ belongs to $L^{\infty}\left(\Omega_{T}\right)$ also when $\mathrm{f}$ is a function not identically zero satisfying (1.12) and

$$
\frac{1}{m}+\frac{N}{p s}<1
$$

Thus, there exists a solution $u$ of (1.1) that becomes immediately bounded also for sufficiently regular data $\mathrm{f}$ (i.e., satisfying (1.16)) even if $u_{0}$ is only a summable function.

We point out that (1.15) holds true for every $u_{0} \in L^{1}(\Omega)$ and for every forcing term satisfying (1.12); hence also if $\mathrm{f}$ does not satisfy (1.16), an improvement in summability immediately appears and again $u(t)$ immediately reaches the same summability of $u^{0}$ (see Corollary 2.2 for further details). To our knowledge, this regularizing phenomenon is not known in the literature.

Moreover, we prove that the following estimate holds true for almost every $t \in(0, T)$

$$
\left\|u(t)-u^{0}(t)\right\|_{L^{\infty}(\Omega)} \leq \min \left\{\frac{C_{0}}{t^{\frac{1}{p-2}}}, C_{1} \frac{\left\|u_{0}\right\|_{L^{1}(\Omega)}^{\frac{p}{N(p-2)+p}}}{t^{\frac{N}{N(p-2)+p}}}\right\},
$$


where $C_{i}, i=0,1$ are positive constants depending only on $\mathrm{p}, \mathrm{N}$ and the coercivity constant $\alpha$ defined in (1.5). Notice that the previous result includes, as particular cases, the decay estimates (1.8) and (1.9), since, as recalled above, when the forcing term $\mathrm{f}$ is identically zero also $u^{0}$ is identically zero.

We notice that the previous estimate is rather surprising since it holds true for every choice of $\mathrm{f}$ and hence, also when the condition (1.16) is not satisfied and hence when generally both $u(t)$ and $u^{0}(t)$ are not in $L^{\infty}(\Omega)$. Thus, the difference between these solutions $u$ and $u^{0}$ is bounded although it may happen that both these solutions are unbounded!

Indeed, we will show that there exists a global solution $u$ of (1.1) (see Definition 2.2) satisfying the bound (1.17) for almost every $t>0$. This last result allows to describe the asymptotic behavior in time of $u(t)$ and implies that

$$
\lim _{t \rightarrow+\infty}\left\|u(t)-u^{0}\right\|_{L^{\infty}(\Omega)}=0 .
$$

In other words, for $t$ large the solution $u(t)$ forgets its "initial value $u_{0}$ " and has the same behavior of the solution $u^{0}$ which satisfies a null condition.

In this paper, we also study the autonomous case:

$$
a(x, t, \nabla u)=a(x, \nabla u) \quad f(x, t)=f(x)
$$

and we prove that for every $u_{0} \in L^{1}(\Omega)$ and $f \in L^{1}(\Omega)$ there exists a global solution $u(t)$ of (1.1) that satisfies

$$
\lim _{t \rightarrow+\infty}\|u(t)-w\|_{L^{\infty}(\Omega)}=0,
$$

where $w$ is the solution (obtained by approximation) of the associate stationary problem

$$
\begin{cases}-\operatorname{div}(a(x, \nabla w))=f(x) & \text { in } \Omega, \\ w=0 & \text { on } \partial \Omega .\end{cases}
$$

In other words, $u(t)$ tends in the $L^{\infty}(\Omega)$-norm to the solution $\mathrm{w}$ of the nonlinear elliptic problem (1.18) even when both these solutions, $u(t)$ and $w$, do not belong to $L^{\infty}(\Omega)$ !

Finally, we investigate what happens to the results described above in the more general case of Radon measures as initial data which is an open problem even in the case of null function f. We complete our investigation considering the case of forcing term $f$ Radon measure too and extending our study also to the semilinear case $p=2$ being this last case not present in the literature (see $[2,8,16,23]$ and the references therein).

We show that most of the results remain true in this more general setting. In particular, the instantaneous regularizing property (1.15) holds true and the $L^{\infty}$-estimate (1.17) described above in the framework of measure data becomes

$$
\left\|u(t)-u^{0}(t)\right\|_{L^{\infty}(\Omega)} \leq \min \left\{\frac{C_{0}}{t^{\frac{1}{p-2}}}, C_{1} \frac{\left\|u_{0}\right\|_{M(\Omega)}^{\frac{p}{N(p-2)+p}}}{t^{\frac{N}{N(p-2)+p}}}\right\} \quad \text { if } p>2
$$

and

$$
\left\|u(t)-u^{0}(t)\right\|_{L^{\infty}(\Omega)} \leq \min \left\{C_{2} \frac{\left\|u_{0}\right\|_{M(\Omega)}}{t^{\frac{N}{2}} e^{\sigma t}}, C_{1} \frac{\left\|u_{0}\right\|_{M(\Omega)}}{t^{\frac{N}{2}}}\right\} \quad \text { if } p=2
$$

where $C_{0}$ and $C_{1}$ are the same positive constants in (1.17) (hence they depend only on $N, p$ and $\alpha$ ), $C_{2}$ is a positive constant depending only on $\mathrm{N}$ and $\alpha$ and $\sigma$ is a positive constant depending only on $\alpha,|\Omega|$ and $N$ (see formula (4.52)). 
The plan of the paper is the following: in Sect. 2 we state all our results. In Sect. 3, for the convenience of the reader, we recall some known results which we use in the proofs. Finally, Sect. 4 is devoted to the proofs of all the results.

\section{Main results}

We state here our results in all the details. We start considering the case of data in Lebesgue spaces and then (in Sect. 2.2) we will study what happens in the presence of measure data.

\subsection{Data in Lebesgue spaces}

Before enouncing our results, we recall what we mean by a solution of (1.1).

Definition 2.1 Assume that the data $\mathrm{f}$ and $u_{0}$ satisfy

$$
f \in L^{1}\left(\Omega_{T}\right) \quad u_{0} \in L^{1}(\Omega) .
$$

Then a measurable function $u \in L^{\infty}\left(0, T ; L^{1}(\Omega)\right) \cap L^{1}\left(0, T ; W_{0}^{1,1}(\Omega)\right)$ is a solution of (1.1) if $a(x, t, \nabla u) \in\left(L^{1}\left(\Omega_{T}\right)\right)^{N}$ and

$$
\iint_{\Omega_{T}}\left[-u \frac{\partial \varphi}{\partial t}+a(x, t, \nabla u) \nabla \varphi\right] \mathrm{d} x \mathrm{~d} t=\int_{\Omega} u_{0} \varphi(0) \mathrm{d} x+\iint_{\Omega_{T}} f \varphi,
$$

for every $\varphi \in W^{1, \infty}\left(0, T ; L^{\infty}(\Omega)\right) \cap L^{\infty}\left(0, T ; W_{0}^{1, \infty}(\Omega)\right)$ satisfying $\varphi(T)=0$.

We observe that under the structure conditions (1.2)-(1.4) and the assumption (2.1) on the data, there exists at least one solution of (1.1) (see [7]). As noticed above, these solutions (in absence of further requirements) are not unique.

One of the main results of this paper is the following $L^{\infty}$-estimate which describes the behavior in time of the distance between two solutions of (1.1) satisfying different initial data in $L^{1}(\Omega)$ and that can be considered as a sort of "continuous dependence of the initial data" (see also Remark 2.1). The importance of this estimate relies not only in the estimate itself, which is rather surprising, but also in various interesting properties of the solutions that it implies and that we will explain below. In detail, let us consider the following nonlinear parabolic equation

$$
\begin{cases}v_{t}-\operatorname{div}(a(x, t, \nabla v))=f(x, t) & \text { in } \Omega_{T}, \\ v=0 & \text { on } \Gamma \\ v(x, 0)=v_{0} & \text { on } \Omega\end{cases}
$$

(i.e., problem (1.1) with initial datum $v_{0}$ ) where

$$
v_{0} \in L^{1}(\Omega) \text {. }
$$

We have the following result

Theorem 2.1 Let (1.2)-(1.4) be satisfied. Assume (2.1) and (2.4). Then there exists at least a solution $u \in C\left([0, T] ; L^{1}(\Omega)\right)$ of $(1.1)$ and there is at least a solution $v \in C\left([0, T] ; L^{1}(\Omega)\right)$ of (2.3) such that the following estimate holds

$$
\|u(t)-v(t)\|_{L^{\infty}(\Omega)} \leq \min \left\{\frac{C_{0}}{t^{\frac{1}{p-2}}}, C_{1} \frac{\left\|u_{0}-v_{0}\right\|_{L^{1}(\Omega)}^{\frac{p}{N(p-2)+p}}}{t^{\frac{N}{N(p-2)+p}}}\right\} \text { for almost every } t \in(0, T)
$$


where $C_{0}$ and $C_{1}$ are positive constants depending only on $N, p$ and $\alpha$.

Moreover, $u$ and $v$ are obtained as the limit (a.e. in $\Omega_{T}$ ) of "classical" solutions of the approximating problems (respectively) (4.1) and (4.4).

Finally, if $u$ and $v$ are two solutions of, respectively (1.1) and (2.3), obtained as the limit (a.e. in $\Omega_{T}$ ) of solutions of the approximating problems (respectively) (4.1) and (4.4), then they satisfy (2.5).

As noticed in Introduction, estimate (2.5) is rather amazing since in general both the solution $u$ and $v$ are not bounded.

We observe that the previous result can be generalized to global solutions. To show the existence of these global solutions, let us assume

$$
h \in L_{l o c}^{p^{\prime}}\left([0,+\infty) ; L^{p^{\prime}}(\Omega)\right)
$$

(where $\mathrm{h}$ is the function in (1.4)) and

$$
f \in L_{l o c}^{1}\left([0,+\infty) ; L^{1}(\Omega)\right), \quad u_{0} \in L^{1}(\Omega) .
$$

The following result holds true.

Theorem 2.2 Let (1.2)-(1.4) and (2.6) be satisfied. Assume (2.7) and (2.4). Then there exists at least a global solution $u \in C_{l o c}\left([0,+\infty) ; L^{1}(\Omega)\right)$ of $(1.1)$ and there is at least a global solution $v \in C_{\text {loc }}\left([0,+\infty) ; L^{1}(\Omega)\right)$ of $(2.3)$ such that the following estimate is satisfied

$$
\|u(t)-v(t)\|_{L^{\infty}(\Omega)} \leq \min \left\{\frac{C_{0}}{t^{\frac{1}{p-2}}}, C_{1} \frac{\left\|u_{0}-v_{0}\right\|_{L^{1}(\Omega)}^{\frac{p}{N(p-2)+p}}}{t^{\frac{N}{N(p-2)+p}}}\right\} \text { for almost every } t>0
$$

where $C_{0}$ and $C_{1}$ are the same constants in (2.5), hence positive constants depending only on $N, p$ and $\alpha$.

In particular, it results

$$
\lim _{t \rightarrow+\infty}\|u(t)-v(t)\|_{L^{\infty}(\Omega)}=0 .
$$

Finally, $u$ and $v$ are obtained as limit of regular approximating solutions.

We recall that here by a global solution we mean the following.

Definition 2.2 A measurable function $u$ is a global solution of (1.1) if it is a solution of (1.1) for every $T>0$ arbitrarily fixed. We also denote a global solution of (1.1) as a solution of the following problem

$$
\begin{cases}u_{t}-\operatorname{div}(a(x, t, \nabla u))=f(x, t) & \text { in } \Omega_{\infty}, \\ u=0 & \text { on } \partial \Omega \times(0,+\infty), \\ u(x, 0)=u_{0}(x) & \text { on } \Omega .\end{cases}
$$

Remark 2.1 We point out that the global solutions $u$ and $v$ constructed in Theorem 2.2 are obtained by approximation. We recall that the solutions in $\Omega_{T}$ constructed by approximation are unique, i.e., if you change the approximation sequences of the data you always get (passing to the limit) the same solution named for this reason "solution obtained by approximation" (see [11]). Hence, the result of Theorem 2.2 is valid not only for the particular solutions constructed here but for all the solutions constructed by approximation. In particular, this implies that all the global solutions constructed by approximation have the same asymptotic 
behavior for $t \rightarrow+\infty$ independently from the initial datum that they satisfy. In other words, the initial data do not influence the behavior for large value of $t$ of all these solutions.

Moreover, a continuous dependence from the initial data holds true for all the solutions of problem (1.1) constructed by approximation.

In the autonomous case

$$
a(x, t, \xi)=a(x, \xi) \quad f(x, t)=f(x)
$$

the previous result imply the following.

Corollary 2.1 Let (1.2)-(1.4), (2.6), (2.7) and (2.11) be satisfied. Then there exists a global solution $u \in C_{l o c}\left([0,+\infty) ; L^{1}(\Omega)\right)$ of (1.1) (that is the unique solution constructed by approximation) such that

$$
\lim _{t \rightarrow+\infty}\|u(t)-w\|_{L^{\infty}(\Omega)}=0,
$$

where $w$ is the (unique) solution of the stationary problem (2.14) obtained by approximation. Moreover, the following estimate is satisfied

$$
\|u(t)-w\|_{L^{\infty}(\Omega)} \leq \min \left\{\frac{C_{0}}{t^{\frac{1}{p-2}}}, C_{1} \frac{\left\|u_{0}-w\right\|_{L^{1}(\Omega)}^{\frac{p}{N(p-2)+p}}}{t^{\frac{N}{N(p-2)+p}}}\right\} \text { for almost every } t>0
$$

where $C_{0}$ and $C_{1}$ are the same constants in (2.5).

Here, by a solution w of

$$
\begin{cases}-\operatorname{div}(a(x, \nabla w))=f(x) & \text { in } \Omega, \\ w=0 & \text { on } \partial \Omega,\end{cases}
$$

we mean a function $w \in W_{0}^{1,1}(\Omega)$ such that $a(x, \nabla w) \in\left(L^{1}(\Omega)\right)^{N}$ and

$$
\int_{\Omega} a(x, \nabla w) \nabla \varphi \mathrm{d} x=\int_{\Omega} f \varphi
$$

for every $\varphi \in W_{0}^{1, \infty}(\Omega)$. Moreover, we say that $\mathrm{w}$ is a solution of (2.14) constructed by approximation if it is obtained as limit in $L^{1}(\Omega)$ of the solutions $w_{n} \in W_{0}^{1, p}(\Omega) \cap L^{\infty}(\Omega)$ of the following approximating problems

$$
\begin{cases}-\operatorname{div}\left(a\left(x, \nabla w_{n}\right)\right)=f_{n}(x) & \text { in } \Omega, \\ w_{n}=0 & \text { on } \partial \Omega,\end{cases}
$$

where $f_{n} \in L^{\infty}(\Omega)$ satisfies

$$
f_{n}(x) \rightarrow f(x) \text { in } L^{1}(\Omega)
$$

Hence, in the autonomous case, whatever is the choice of the initial datum $u_{0}$, the "corresponding" global solution of (1.1) which take $u_{0}$ as initial datum and is obtained by approximation, converges (letting $t \rightarrow+\infty$ ) to the (unique) solution of the associate stationary problem (2.14) obtained by approximation. We show now that the estimates proved in the previous theorems allow to prove also interesting information on the regularity of the solutions of (1.1). 
To this aim, let $u^{0}$ be as in Introduction the (unique) solution of (1.1) constructed by approximation which assume zero initial datum. Thus, $u^{0}$ solves (1.14) and is the a.e. limit in $\Omega_{T}$ of the regular solutions $u_{n}^{0}$ of the following approximating problems

$$
\begin{cases}\left(u_{n}^{0}\right)_{t}-\operatorname{div}\left(a\left(x, t, \nabla u_{n}^{0}\right)\right)=f_{n}(x, t) & \text { in } \Omega_{T}, \\ u_{n}^{0}=0 & \text { on } \Gamma \\ u_{n}^{0}(x, 0)=0 & \text { on } \Omega,\end{cases}
$$

where

$$
f_{n} \in L^{\infty}\left(\Omega_{T}\right) \quad f_{n} \rightarrow f \quad \text { in } \quad L^{1}\left(\Omega_{T}\right)
$$

Recall that under the assumptions of Theorem 2.2 (i.e., (1.2)-(1.4), (2.6) and $\mathrm{f}$ as in (2.7)) $u^{0}$ has a global extension in $\Omega \times(0,+\infty)$.

We notice explicitly that in all what follows the solution $u^{0}$ can be replaced by any solution $v$ of (2.3) (constructed by approximation) that satisfy a bounded initial condition.

Theorem 2.3 Assume (1.2)-(1.4) and (2.1). Let $u^{0}$ be the solution of (1.14) defined above. Then there exists a solution $u \in C\left([0, T] ; L^{1}(\Omega)\right)$ of $(1.1)$ which in every set $\Omega \times(\varepsilon, T)$, $\varepsilon \in(0, T)$, has the same summability properties of $u^{0}$, i.e., for every $\varepsilon \in(0, T)$

$$
u^{0} \in L^{\delta}\left(\varepsilon, T ; L^{v}(\Omega)\right) \Longrightarrow u \in L^{\delta}\left(\varepsilon, T ; L^{v}(\Omega)\right)
$$

where $\delta$ and $v$ belong to the set $[1,+\infty]$. In particular, it results

$$
u^{0} \in L^{\delta}\left(0, T ; L^{\nu}(\Omega)\right) \Longrightarrow u \in L_{l o c}^{\delta}\left((0, T] ; L^{\nu}(\Omega)\right)
$$

Moreover, the following estimate holds true for every $\varepsilon \in(0, T)$

$$
\|u\|_{L^{\delta}\left(\varepsilon, T ; L^{\nu}(\Omega)\right)} \leq\left\|u^{0}\right\|_{L^{\delta}\left(\varepsilon, T ; L^{v}(\Omega)\right)}+\min \left\{\frac{C_{0}}{\varepsilon^{\frac{1}{p-2}}}, C_{1} \frac{\left\|u_{0}\right\|_{L^{1}(\Omega)}^{\frac{p}{N(p-2)+p}}}{\varepsilon^{\frac{N}{N(p-2)+p}}}\right\}|\Omega|^{\frac{1}{v}}(T-\varepsilon)^{\frac{1}{\delta}}
$$

with $C_{0}$ and $C_{1}$ as in (2.5), and where (here and throughout the paper) we adopt the convention that $\frac{1}{+\infty}=0$ and we denote $|\Omega|$ the measure of $\Omega$. In particular, the following universal estimate is satisfied

$$
\|u\|_{L^{\delta}\left(\varepsilon, T ; L^{\nu}(\Omega)\right)} \leq\left\|u^{0}\right\|_{L^{\delta}\left(\varepsilon, T ; L^{\nu}(\Omega)\right)}+\frac{C_{0}}{\varepsilon^{\frac{1}{p-2}}}|\Omega|^{\frac{1}{v}}(T-\varepsilon)^{\frac{1}{\delta}} .
$$

Finally, $u$ is obtained as the limit (a.e. in $\Omega_{T}$ ) of the approximating solutions $u_{n}$ of problem (4.1) and under the further "global assumptions" (2.6) and (2.7) it can be extended to a global solution (denoted again u) satisfying the assert of Theorem 2.2.

Thus, thanks to the previous result, we can assert that, although the initial datum $u_{0}$ is only a summable function, instantaneously the solution $u$ reaches the same regularity of the solution $u^{0}$ which satisfy a bounded initial datum condition. Moreover, for every $\varepsilon>0$ arbitrarily fixed, it is possible to estimate the norm of $u$ in every set $\Omega \times(\varepsilon, T)$ with a constant independent of $u_{0}$. Universal estimates are known in the literature when $f \equiv 0$ and in the framework of bounded solutions. Hence, estimate (2.21) allows to conclude that universal estimates remain true also for nonzero data $\mathrm{f}$ and for possibly unbounded solutions.

An immediate consequence of Theorem 2.3 is the following regularity result. 
Corollary 2.2 Assume (1.2)-(1.4), $u_{0} \in L^{1}(\Omega)$ and $f \in L^{m}\left(0, T ; L^{s}(\Omega)\right)$ with $m$ and $s$ in $(1,+\infty]$. Then there exists a solution $u \in C\left([0, T] ; L^{1}(\Omega)\right)$ of $(1.1)$ satisfying, for every $\varepsilon>0$, the following regularity properties

$$
\begin{aligned}
& u \in L^{\infty}(\Omega \times(\varepsilon, T)) \text { if } \frac{1}{m}+\frac{N}{p s}<1 \\
& u \in L^{q}(\Omega \times(\varepsilon, T)) \quad \text { if } 1<\frac{1}{m}+\frac{N}{p s} \leq \frac{N}{p m}+1, \quad m \geq p^{\prime}, \quad s>1, \\
& u \in L^{q}(\Omega \times(\varepsilon, T)) \quad \text { if } 1<\frac{1}{m}+\frac{N}{p s} \leq \frac{N}{p m}+1+\frac{N}{2}\left(1-\frac{1}{p}-\frac{1}{m}\right), \quad 1 \leq m<p^{\prime}, \quad s \geq 1
\end{aligned}
$$

where

$$
q=\frac{s m(N+p)+N(p-2)(m s-s+m)}{N m-p r(m-1)}
$$

Indeed, there is "an immediate improvement in regularity" also of the gradient of $u$ if the datum $\mathrm{f}$ is not too irregular. In detail, it results:

Theorem 2.4 Let (1.2)-(1.4) be satisfied. Assume $u_{0} \in L^{1}(\Omega)$ and $f \in L^{m}\left(0, T ; L^{s}(\Omega)\right)$ with $m$ and $s$ in $(1,+\infty]$ satisfying

$$
\frac{1}{m}+\frac{N}{p s}<1
$$

Then there exists a solution u of (1.1) (which is the same solution that appears in Theorems 2.1 and 2.2 ) satisfying

$$
u \in L^{\infty}\left(\varepsilon, T ; L^{\infty}(\Omega)\right) \cap C\left([0, T] ; L^{1}(\Omega)\right) \cap L^{p}\left(\varepsilon, T ; W_{0}^{1, p}(\Omega)\right)
$$

for every $0<\varepsilon<T$ and verifying the following estimates for almost every $t \in(0, T)$

$$
\|u(t)\|_{L^{\infty}(\Omega)} \leq A(t) \equiv c_{0}\|f\|_{L^{m}\left(0, T ; L^{s}(\Omega)\right)}+\min \left\{\frac{C_{0}}{t^{\frac{1}{p-2}}}, C_{1} \frac{\left\|u_{0}\right\|_{L^{1}(\Omega)}^{\frac{p}{N(p-2)+p}}}{t^{\frac{N}{N(p-2)+p}}}\right\},
$$

and

$$
\int_{t}^{T} \int_{\Omega}|\nabla u|^{p} \leq \frac{1}{\alpha} A(t)\left(\frac{|\Omega|}{2} A(t)+\|f\|_{L^{1}\left(\Omega_{T}\right)}\right)
$$

where $c_{0}=c_{0}(T,|\Omega|, N, \alpha)$ and $C_{0}$ and $C_{1}$ are exactly the same constants that appear in (2.5). In particular, the following universal estimates hold true

$$
\begin{gathered}
\|u(t)\|_{L^{\infty}(\Omega)} \leq B(t) \equiv c_{0}\|f\|_{L^{m}\left(0, T ; L^{s}(\Omega)\right)}+\frac{C_{0}}{t^{\frac{1}{p-2}}}, \\
\int_{t}^{T} \int_{\Omega}|\nabla u|^{p} \leq \frac{1}{\alpha} B(t)\left(\frac{|\Omega|}{2} B(t)+\|f\|_{L^{1}\left(\Omega_{T}\right)}\right) .
\end{gathered}
$$

Moreover, the solution $u$ is obtained as the limit (a.e. in $\Omega_{T}$ ) of the approximating solutions $u_{n}$ of (4.1). Finally, $u$ is Hoelder continuous (of exponent $\beta$ with respect to $x$ and $\frac{\beta}{p}$ with respect to $t)$ in $\bar{\Omega} \times(\varepsilon, T)$, for every $0<\varepsilon<T$. In particular, it results

$$
u \in C(\bar{\Omega} \times(0, T)) \cap C\left((0, T) ; L^{2}(\Omega)\right) .
$$

Remark 2.2 We notice that the previous result reveals that although the initial datum $u_{0}$ is only in $L^{1}(\Omega)$ there exists a solution $u$ which becomes immediately bounded and whose 
gradient increases its summability too. Moreover, universal estimates, i.e., estimate which are not influenced at all by the initial datum $u_{0}$ are satisfied both by the $L^{\infty}$-norm of $u$ and by the $L^{p}$-norm of the gradient of $\mathrm{u}$. To our knowledge these universal estimates are not known in the literature for nonzero data $\mathrm{f}$.

Remark 2.3 We recall that it is well known that if $\mathrm{f}$ belongs to $L^{m}\left(0, T ; L^{S}(\Omega)\right)$ with $\mathrm{m}$ and $\mathrm{s}$ satisfying (2.22), also without any information on the initial datum $u_{0}$, all the solution of (1.1) belonging to $C\left(0, T ; L^{2}(\Omega)\right) \cap L^{p}\left(0, T ; W_{0}^{1, p}(\Omega)\right)$ are bounded (see [12] and the references therein). Moreover, the result has a local nature, i.e., if $u$ is a local solution belonging to $C_{l o c}\left(0, T ; L_{l o c}^{2}(\Omega)\right) \cap L_{l o c}^{p}\left(0, T ; W_{l o c}^{1, p}(\Omega)\right)$ and $\mathrm{f}$ is in $L_{l o c}^{m}\left(0, T ; L_{l o c}^{s}(\Omega)\right)$ (with $\mathrm{m}$ and $\mathrm{s}$ as before) then $\mathrm{u}$ is locally bounded (see [12,17] and the references therein). The difference here with the classical $L^{\infty}$-theory is that the "starting regularity" $C\left(0, T ; L^{2}(\Omega)\right) \cap$ $L^{p}\left(0, T ; W_{0}^{1, p}(\Omega)\right)$ is not assumed since we do not know if solutions with $L^{1}$-initial data have such a regularity. Moreover, the $L^{\infty}$-estimates that we derive are completely different from the classical ones since they do not depend at all from the solution itself.

Indeed, even if the initial datum is only a summable function, it is sufficient that $f$ belongs to the space $L^{p^{\prime}}\left(0, T ; W^{-1, p^{\prime}}(\Omega)\right)$ to have solutions that immediately belong to the energy space $C\left((0, T) ; L^{2}(\Omega)\right) \cap L_{l o c}^{p}\left(0, T ; W_{0}^{1, p}(\Omega)\right)$ as the following result shows.

Theorem 2.5 Let (1.2)-(1.4) be satisfied. Assume $u_{0} \in L^{1}(\Omega)$ and $f \in L^{p^{\prime}}\left(0, T ; W^{-1, p^{\prime}}\right.$ $(\Omega)$ ), i.e., $f=f_{0}-\operatorname{div}\left(f_{1}\right)$, with $f_{i} \in L^{p^{\prime}}\left(\Omega_{T}\right), i=0,1$. Then there exists a solution ${ }^{2}$ $u$ of $(1.1)$ belonging to $C\left([0, T] ; L^{1}(\Omega)\right) \cap C\left((0, T) ; L^{2}(\Omega)\right) \cap L_{l o c}^{p}\left(0, T ; W_{0}^{1, p}(\Omega)\right)^{3}$ and satisfying the following estimates

$$
\begin{aligned}
& \|u\|_{L^{\infty}\left(\varepsilon, T ; L^{2}(\Omega)\right)} \leq C_{\alpha}\left[\left\|f_{0}\right\|_{L^{p^{\prime}}\left(\Omega_{T}\right)}+\left\|f_{1}\right\|_{L^{p^{\prime}}\left(\Omega_{T}\right)}\right]^{\frac{p^{\prime}}{2}} \\
& +\min \left\{\frac{C_{0}}{\varepsilon^{\frac{1}{p-2}}}, C_{1} \frac{\left\|u_{0}\right\|_{L^{1}(\Omega)}^{\frac{p}{N(p-2)+p}}}{\varepsilon^{\frac{N}{N(p-2)+p}}}\right\}|\Omega|^{\frac{1}{2}}, \\
& \int_{t}^{T} \int_{\Omega}|\nabla u|^{p} \leq c_{0}\left[\left\|f_{0}\right\|_{L^{p^{\prime}}\left(\Omega_{T}\right)}+\left\|f_{1}\right\|_{L^{p^{\prime}}\left(\Omega_{T}\right)}\right]^{p^{\prime}} \\
& +\frac{p^{\prime}}{\alpha} \min \left\{\frac{C_{0}^{2}}{t^{\frac{2}{p-2}}}, C_{1}^{2} \frac{\left\|u_{0}\right\|_{L^{1}(\Omega)}^{\frac{2 p}{N(2)+p}}}{t^{\frac{2 N}{N(p-2)+p}}}\right\}|\Omega|, \quad \text { for every } 0<t \leq T,
\end{aligned}
$$

where $C_{\alpha}=\left[\frac{2}{p^{\prime} \alpha^{\frac{1}{p-1}}}\left(\max \left\{1, c_{P}\right\}\right)^{p^{\prime}}\right]^{\frac{1}{2}}, c_{P}$ is the Poincaré constant defined in (4.36) and $c_{0}=\frac{p^{\prime}}{\alpha} C_{\alpha}^{2}+\frac{1}{\alpha^{p^{\prime}}}\left(\max \left\{1, c_{P}\right\}\right)^{p^{\prime}}$.

${ }^{2}$ Here, similarly to Definition 2.1 , by a solution of (1.1) we mean a measurable function $u \in$ $L^{\infty}\left(0, T ; L^{1}(\Omega)\right) \cap L^{1}\left(0, T ; W_{0}^{1,1}(\Omega)\right)$ such that $a(x, t, \nabla u) \in\left(L^{1}\left(\Omega_{T}\right)\right)^{N}$ and

$$
\iint_{\Omega_{T}}\left[-u \frac{\partial \varphi}{\partial t}+a(x, t, \nabla u) \nabla \varphi\right] \mathrm{d} x \mathrm{~d} t=\int_{\Omega} u_{0} \varphi(0) \mathrm{d} x+\iint_{\Omega_{T}}\left[f_{0} \varphi+f_{1} \nabla \varphi\right],
$$

for every $\varphi \in W^{1, \infty}\left(0, T ; L^{\infty}(\Omega)\right) \cap L^{\infty}\left(0, T ; W_{0}^{1, \infty}(\Omega)\right)$ satisfying $\varphi(T)=0$.

${ }^{3}$ By $L_{l o c}^{p}\left(0, T ; W_{0}^{1, p}(\Omega)\right)$ we mean the space $L_{l o c}^{p}\left((0, T] ; W_{0}^{1, p}(\Omega)\right)$. 
In particular the following universal estimates hold true

$$
\begin{gathered}
\|u\|_{L^{\infty}\left(\varepsilon, T ; L^{2}(\Omega)\right)} \leq C_{\alpha}\left[\left\|f_{0}\right\|_{L^{p^{\prime}\left(\Omega_{T}\right)}}+\left\|f_{1}\right\|_{L^{p^{\prime}\left(\Omega_{T}\right)}}\right]^{\frac{p^{\prime}}{2}}+\frac{C_{0}}{\varepsilon^{\frac{1}{p-2}}|\Omega|^{\frac{1}{2}},} \quad, \quad \text { for every } 0<t \leq T, \\
\int_{t}^{T} \int_{\Omega}|\nabla u|^{p} \leq c_{0}\left[\left\|f_{0}\right\|_{L^{p^{\prime}}\left(\Omega_{T}\right)}+\left\|f_{1}\right\|_{L^{p^{\prime}}\left(\Omega_{T}\right)}\right]^{p^{\prime}}+\frac{c_{1}}{t^{\frac{2}{p-2}}}, \quad \text { for }
\end{gathered}
$$

where $c_{1}=\frac{C_{0}^{2} p^{\prime}}{\alpha}|\Omega|$. Finally, $u$ is the same solution satisfying Theorems $2.1-2.3$ and hence it is obtained as the limit (a.e. in $\Omega_{T}$ ) of the regular solution $u_{n}$ of (4.1).

We prove now that under the assumptions of the previous theorem, the solutions that immediately regularize are unique. In detail we have the following result.

Theorem 2.6 Under the assumptions of Theorem 2.5 there exists only one solution $u$ of (1.1) belonging to $C\left([0, T] ; L^{1}(\Omega)\right) \cap C\left((0, T) ; L^{2}(\Omega)\right) \cap L_{l o c}^{p}\left(0, T ; W_{0}^{1, p}(\Omega)\right)$.

Moreover, if $v$ is a solution of (2.3) with $v_{0} \in L^{1}(\Omega)$ and if $u$ is a solution of (1.1) and if both $u$ and $v$ belong to $C\left([0, T] ; L^{1}(\Omega)\right) \cap C\left((0, T) ; L^{2}(\Omega)\right) \cap L_{l o c}^{p}\left(0, T ; W_{0}^{1, p}(\Omega)\right)$ then the following estimate ("continuous dependence from the initial data") holds true

$$
\|u(t)-v(t)\|_{L^{\infty}(\Omega)} \leq C_{1} \frac{\left\|u_{0}-v_{0}\right\|_{L^{1}(\Omega)}^{\frac{p}{N(p-2)+p}}}{t^{\frac{N}{N(p-2)+p}}} \text { for almost every } t \in(0, T)
$$

where $C_{1}$ is the same constant (depending only on $N, p$ and $\alpha$ ) that appears in (2.5).

Remark 2.4 An immediate consequence of the previous result together with Theorem 2.5 (see also Remark 4.2) is that the solutions constructed by approximation are unique. We point out that it is well known in the literature that the solutions constructed by approximation are unique (and also in a more general setting) but here the proof of this result is completely different and shows that indeed the uniqueness holds in the set of the solutions that immediately increase their regularity. Thus, the "reason" of the uniqueness of the solutions constructed by approximation is their regularity $C\left([0, T] ; L^{1}(\Omega)\right) \cap C\left((0, T) ; L^{2}(\Omega)\right) \cap$ $L_{l o c}^{p}\left(0, T ; W_{0}^{1, p}(\Omega)\right)$.

We conclude this section showing (in the following subsection) that most of the previous results remain true in the more general framework of Radon measure data.

\subsection{Measure data}

Let us denote $M(O)$ the set of (finite) Radon measures on $O$. As usual, if $\mathrm{f}$ is a Radon measure on $O$, we identify the measure $\mathrm{f}$ with the application $\varphi \rightarrow \int_{O} \varphi \mathrm{d} f \operatorname{defined}$ on $C(\bar{O})$. If we assume

$$
u_{0} \in M(\Omega)
$$

and

$$
f \in M\left(\Omega_{T}\right)
$$

by a solution of (1.1) we mean the following 
Definition 2.3 Under the assumptions (2.35) and (2.36) a function $u \in L^{1}\left(0, T ; W_{0}^{1,1}(\Omega)\right)$ is a solution of (1.1) if $a(x, t, \nabla u) \in\left(L^{1}\left(\Omega_{T}\right)\right)^{N}$ and

$$
\iint_{\Omega_{T}}\left[-u \frac{\partial \varphi}{\partial t}+a(x, t, \nabla u) \nabla \varphi\right] \mathrm{d} x \mathrm{~d} t=\int_{\Omega} \varphi(0) d u_{0}+\iint_{\Omega_{T}} \varphi \mathrm{d} f,
$$

for every $\varphi \in C^{\infty}\left(\overline{\Omega_{T}}\right)$ which is zero in a neighborhood of $\Gamma \cup(\Omega \times\{T\})$.

As noticed above, here we investigate both the semilinear case $p=2$ and the degenerate case $p>2$. The singular case $p<2$ will be the subject of a forthcoming paper (see [26]). We recall that if the data $u_{0}$ and $f$ are finite Radon measures and if the structure conditions (1.2)-(1.4) are satisfied with $p \geq 2$, then there exists at least one solution of (1.1) (see [7]). We have the following results:

Theorem 2.7 Let (1.2)-(1.4) be satisfied with $p \geq 2$. Assume (2.35), (2.36) and $v_{0}$ in $M(\Omega)$. Then there exist $u$ and $v$ solutions of, respectively, (1.1) and of (2.3) such that the following estimates hold true for almost every $t \in(0, T)$

$$
\|u(t)-v(t)\|_{L^{\infty}(\Omega)} \leq \min \left\{\frac{C_{0}}{t^{\frac{1}{p-2}}}, C_{1} \frac{\left\|u_{0}-v_{0}\right\|_{M(\Omega)}^{\frac{p}{N(p-2)+p}}}{t^{\frac{N}{N(p-2)+p}}}\right\} \quad \text { if } p>2
$$

and

$$
\|u(t)-v(t)\|_{L^{\infty}(\Omega)} \leq \min \left\{C_{2} \frac{\left\|u_{0}-v_{0}\right\|_{M(\Omega)}}{t^{\frac{N}{2}} e^{\sigma t}}, C_{1} \frac{\left\|u_{0}-v_{0}\right\|_{M(\Omega)}}{t^{\frac{N}{2}}}\right\} \quad \text { if } p=2
$$

where $C_{0}$ and $C_{1}$ are the same positive constants defined in (2.5), $C_{2}$ is a positive constant depending only on $N$ and $\alpha$ and $\sigma$ is a positive constant depending only on $\alpha,|\Omega|$ and $N$ (see formula (4.52)).

Moreover, these solutions $u$ and $v$ are obtained as the limit (a.e. in $\Omega_{T}$ ) of "classical" solutions of the approximating problems (respectively) (4.46) and (4.49).

As already noticed in the case of $L^{1}$-data, the previous estimates are rather unexpected being the data only bounded Radon measures. We point out that in the particular case

$$
f \equiv 0
$$

choosing $v_{0}=0$, by the previous Theorem it follows that there exists a solution $u$ of (1.1) satisfying the following estimates for almost every $t \in(0, T)$

$$
\|u(t)\|_{L^{\infty}(\Omega)} \leq \min \left\{\frac{C_{0}}{t^{\frac{1}{p-2}}}, C_{1} \frac{\left\|u_{0}\right\|_{M(\Omega)}^{\frac{p}{N(p-2)+p}}}{t^{\frac{N}{N(p-2)+p}}}\right\} \quad \text { if } p>2
$$

and

$$
\|u(t)\|_{L^{\infty}(\Omega)} \leq \min \left\{C_{2} \frac{\left\|u_{0}\right\|_{M(\Omega)}}{t^{\frac{N}{2}} e^{\sigma t}}, C_{1} \frac{\left\|u_{0}\right\|_{M(\Omega)}}{t^{\frac{N}{2}}}\right\} \quad \text { if } p=2
$$

(being in this case $v \equiv 0$ ). To our knowledge, estimates (2.40) and (2.41) seem not known in literature and show that the $L^{\infty}$-regularization property, well known (when $f \equiv 0$ ) for $L^{1}$-initial data, appears also for measure initial data. Notice that if $p>2$ by (2.40) it follows the universal estimate

$$
\|u(t)\|_{L^{\infty}(\Omega)} \leq \frac{C_{0}}{t^{\frac{1}{p-2}}} \quad \text { for almost every } t \in(0, T)
$$


and hence again, as in the case of initial data in Lebesgue spaces, the $L^{\infty}$ norm of the solution can be estimate by quantity independent of the initial datum itself.

We show now that also in the case of measure data there exist global solutions (according to Definition 2.2) having the same asymptotic behavior in time. We point out that the proof of the existence of such global solutions is different from the previous case of $L^{1}$-data since now the solutions in $\Omega_{T}$ (generally) do not belong to $C\left([0, T] ; L^{1}(\Omega)\right.$ ). In detail, we have:

Theorem 2.8 Let (1.2)-(1.4) and (2.6) be satisfied with $p \geq 2$. Assume (2.35) and $v_{0}$ in $M(\Omega)$. If (2.36) holds for every $T>0$, then there exist a global solution $u$ of (1.1) and a global solution $v$ of (2.3) such that estimate (2.38) if $p>2$ and (2.39) if $p=2$ are satisfied for almost every $t>0$.

In particular, the limit (2.9) holds true, i.e.,

$$
\lim _{t \rightarrow+\infty}\|u(t)-v(t)\|_{L^{\infty}(\Omega)}=0 .
$$

Corollary 2.3 (Autonomous case) Let (2.11), (1.2)-(1.4) and (2.6) be satisfied with $p \geq 2$. Assume (2.35) and $f$ in $M(\Omega)$. Then there exists a global solution $u$ of (1.1) such that for almost every $t>0$

$$
\|u(t)-w\|_{L^{\infty}(\Omega)} \leq \min \left\{\frac{C_{0}}{t^{\frac{1}{p-2}}}, C_{1} \frac{\left\|u_{0}-w\right\|_{M(\Omega)}^{\frac{p}{N(p-2)+p}}}{t^{\frac{N}{N(p-2)+p}}}\right\} \quad \text { if } p>2
$$

and

$$
\|u(t)-w\|_{L^{\infty}(\Omega)} \leq \min \left\{C_{2} \frac{\left\|u_{0}-w\right\|_{M(\Omega)}}{t^{\frac{N}{2}} e^{\sigma t}}, C_{1} \frac{\left\|u_{0}-w\right\|_{M(\Omega)}}{t^{\frac{N}{2}}}\right\} \quad \text { if } p=2
$$

where $C_{0}$ and $C_{1}$ are the same positive constants defined in (2.5), $C_{2}$ and $\sigma$ are the same positive constants defined in (2.39) and $w$ is a solution (constructed by approximation) of the associated stationary problem (2.14). In particular, it results

$$
\lim _{t \rightarrow+\infty}\|u(t)-w\|_{L^{\infty}(\Omega)}=0 .
$$

Here, by a solution $w$ of the stationary problem (2.14) with datum $f$ in $M(\Omega)$ we mean a function $w \in W_{0}^{1,1}(\Omega)$ such that $a(x, \nabla w) \in\left(L^{1}(\Omega)\right)^{N}$ and

$$
\int_{\Omega} a(x, \nabla w) \nabla \varphi \mathrm{d} x=\int_{\Omega} \varphi \mathrm{d} f
$$

for every $\varphi \in C_{0}^{\infty}(\Omega)$.

We point out that the assumptions of Corollary 2.3 guarantee the existence of a solution w of (2.14) (see [7]).

The following result shows that also in the case of measure initial data there exists at least a solution which "immediately" improves its regularity reaching the same regularity of the solutions verifying bounded initial data. In details, let us assume

$$
v_{0} \in L^{\infty}(\Omega) \text { and } f \in M\left(\Omega_{T}\right)
$$

and let $v$ a solution of (2.3) obtained as limit (a.e. in $\Omega_{T}$ ) of $v_{n} \in L^{\infty}\left(\Omega_{T}\right) \cap$ $C\left([0, T] ; L^{2}(\Omega)\right) \cap L^{p}\left(0, T ; W_{0}^{1, p}(\Omega)\right)$ solutions of

$$
\begin{cases}\left(v_{n}\right)_{t}-\operatorname{div}\left(a\left(x, t, \nabla v_{n}\right)\right)=f_{n}(x, t) & \text { in } \Omega_{T}, \\ v_{n}=0 & \text { on } \Gamma \\ v(x, 0)=v_{0} & \text { on } \Omega\end{cases}
$$


where

$f_{n} \in C_{0}^{\infty}\left(\Omega_{T}\right): \quad f_{n}$ converges to $f$ in the weak- * topology of measures.

We recall that under the assumptions (1.2)-(1.4), there exists such a solution $v$ (see [7]). Moreover, in this more general setting of measure, we do not know if the solutions of (2.3) obtained by approximation are unique. Anyway, we have the following regularity result:

Theorem 2.9 Let (1.2)-(1.4) be satisfied with $p \geq 2$. Assume (2.45) and (2.35). If $v$ is as above a solution of (2.3) constructed by approximation, then there exists a solution $u$ of (1.1) having the same summability properties of $v$, i.e., for every $\varepsilon \in(0, T)$ it results

$$
v \in L^{\delta}\left(\varepsilon, T ; L^{v}(\Omega)\right) \quad \Longrightarrow u \in L^{\delta}\left(\varepsilon, T ; L^{v}(\Omega)\right)
$$

where $\delta$ and $v$ belong to the set $[1,+\infty]$. In particular, it results

$$
v \in L^{\delta}\left(0, T ; L^{\nu}(\Omega)\right) \quad \Longrightarrow \quad u \in L_{l o c}^{\delta}\left((0, T] ; L^{\nu}(\Omega)\right)
$$

Moreover, if $p>2$ the following estimate holds true for every $\varepsilon \in(0, T)$

$\|u\|_{L^{\delta}\left(\varepsilon, T ; L^{\nu}(\Omega)\right)} \leq\|v\|_{L^{\delta}\left(\varepsilon, T ; L^{\nu}(\Omega)\right)}+\min \left\{\frac{C_{0}}{\varepsilon^{\frac{1}{p-2}}}, C_{1} \frac{\left\|u_{0}-v_{0}\right\|_{M(\Omega)}^{\frac{p}{N(p-2)+p}}}{\varepsilon^{\frac{N}{N(p-2)+p}}}\right\}|\Omega|^{\frac{1}{v}}(T-\varepsilon)^{\frac{1}{\delta}}$

with $C_{0}$ and $C_{1}$ as in (2.5) (positive constants depending only on $p, N$ and $\alpha$ ). In particular, the following universal estimate holds

$$
\|u\|_{L^{\delta}\left(\varepsilon, T ; L^{v}(\Omega)\right)} \leq\|v\|_{L^{\delta}\left(\varepsilon, T ; L^{\nu}(\Omega)\right)}+\frac{C_{0}}{\varepsilon^{\frac{1}{p-2}}}|\Omega|^{\frac{1}{v}}(T-\varepsilon)^{\frac{1}{\delta}} .
$$

If otherwise $p=2$, for every $\varepsilon \in(0, T)$ it results

$$
\begin{aligned}
& \|u\|_{L^{\delta}\left(\varepsilon, T ; L^{v}(\Omega)\right)} \leq\|v\|_{L^{\delta}\left(\varepsilon, T ; L^{v}(\Omega)\right)} \\
& +\min \left\{C_{2} \frac{\left\|u_{0}-v_{0}\right\|_{M(\Omega)}}{\varepsilon^{\frac{N}{2}} e^{\sigma \varepsilon}}, C_{1} \frac{\left\|u_{0}-v_{0}\right\|_{M(\Omega)}}{\varepsilon^{\frac{N}{2}}}\right\}|\Omega|^{\frac{1}{v}}(T-\varepsilon)^{\frac{1}{\delta}}
\end{aligned}
$$

with $C_{2}$ and $\sigma$ as in (2.39). Finally, $u$ is obtained by approximation.

Hence, an interesting consequence of the previous result is that if $\mathrm{f}$ is in $L^{m}\left(0, T ; L^{s}(\Omega)\right)$ with $\mathrm{m}$ and s satisfying (2.22) and $u_{0}$ is in $M(\Omega)$, then there exists a solution of (1.1) which immediately becomes bounded, despite the fact that the initial datum is only a Radon measure. The following result shows that, analogously to the case of summable initial data, also the gradient of the solution improves instantaneously its regularity.

Theorem 2.10 Let (1.2)-(1.4) be satisfied with $p \geq 2$. Assume $u_{0} \in M(\Omega)$ and $f \in$ $L^{m}\left(0, T ; L^{s}(\Omega)\right)$ with $m$ and $s$ in $(1,+\infty]$ satisfying $(2.22)$. Then there exists a solution $u$ of (1.1) satisfying

$$
u \in L^{\infty}\left(\varepsilon, T ; L^{\infty}(\Omega)\right) \cap L^{p}\left(\varepsilon, T ; W_{0}^{1, p}(\Omega)\right)
$$

for every $0<\varepsilon<T$. Moreover, for almost every $t \in(0, T)$ the following estimates hold true

$$
\begin{aligned}
\|u(t)\|_{L^{\infty}(\Omega)} & \leq A(t), \\
\int_{t}^{T} \int_{\Omega}|\nabla u|^{p} & \leq \frac{1}{\alpha} A(t)\left(\frac{|\Omega|}{2} A(t)+\|f\|_{L^{1}\left(\Omega_{T}\right)}\right)
\end{aligned}
$$


where

$$
\mathrm{A}(t)=c_{0}\|f\|_{L^{m}\left(0, T ; L^{s}(\Omega)\right)}+\min \left\{\frac{C_{0}}{t^{\frac{1}{p-2}}}, C_{1} \frac{\left\|u_{0}\right\|_{M(\Omega)}^{\frac{p}{N(p-2)+p}}}{t^{\frac{N}{N(p-2)+p}}}\right\} \quad \text { if } p>2
$$

and

$$
A(t)=c_{0}\|f\|_{L^{m}\left(0, T ; L^{s}(\Omega)\right)}+\min \left\{C_{2} \frac{\left\|u_{0}\right\|_{M(\Omega)}}{t^{\frac{N}{2}} e^{\sigma t}}, C_{1} \frac{\left\|u_{0}\right\|_{M(\Omega)}}{t^{\frac{N}{2}}}\right\} \quad \text { if } p=2,
$$

with $c_{0}=c_{0}(T,|\Omega|, N, \alpha)$ as in (2.24) and $C_{i}(i=0,1,2)$ and $\sigma$ as in Theorem 2.7.

In particular, if $p>2$ the universal estimates (2.26) and (2.27) are satisfied.

Finally $u$ is Hoelder continuous in $\bar{\Omega} \times(\varepsilon, T)$ and the regularity property (2.28) holds.

An immediate consequence of the previous theorem is the following interesting result.

Corollary 2.4 Let (1.2)-(1.4) be satisfied with $p \geq 2$. Assume $u_{0} \in M(\Omega)$. Then there exists a solution $u$ of $(1.10)$ belonging to $L^{\infty}\left(\varepsilon, T ; L^{\infty}(\Omega)\right) \cap L^{p}\left(\varepsilon, T ; W_{0}^{1, p}(\Omega)\right) \cap C\left([\varepsilon, T] ; L^{2}(\Omega)\right)$ and Holder continuous in $\bar{\Omega} \times(\varepsilon, T)$, for every $\varepsilon \in(0, T)$. Moreover, the following universal decay estimates hold if $p>2$

$$
\|u(t)\|_{L^{\infty}(\Omega)} \leq \frac{C_{0}}{t^{\frac{1}{p-2}}}, \quad \int_{t}^{T} \int_{\Omega}|\nabla u|^{p} \leq \frac{C_{3}}{t^{\frac{2}{p-2}}}
$$

and the following exponential decay estimates are true if $p=2$

$$
\|u(t)\|_{L^{\infty}(\Omega)} \leq C_{2} \frac{\left\|u_{0}\right\|_{M(\Omega)}}{t^{\frac{N}{2}} e^{\sigma t}}, \quad\left(\int_{t}^{T} \int_{\Omega}|\nabla u|^{2}\right)^{\frac{1}{2}} \leq C_{4} \frac{\left\|u_{0}\right\|_{M(\Omega)}}{t^{\frac{N}{2}} e^{\sigma t}}
$$

where $C_{3}=\frac{C_{0}^{2}|\Omega|}{2 \alpha}, C_{4}=\frac{C_{2} \sqrt{|\Omega|}}{\sqrt{2 \alpha}}$ with $C_{0}, C_{2}$ and $\sigma$ as in Theorem 2.7.

Indeed, although the initial data are only Radon measures, to have an immediate improvement in the regularity of the gradient of the solutions (again as in the case of summable initial data) it is sufficient that the data $\mathrm{f}$ belong to $L^{p^{\prime}}\left(0, T ; W^{-1, p^{\prime}}(\Omega)\right)$, as the following result clarify.

Theorem 2.11 Let (1.2)-(1.4) be satisfied with $p \geq 2$. Assume $u_{0} \in M(\Omega)$ and $f \in$ $L^{p^{\prime}}\left(0, T ; W^{-1, p^{\prime}}(\Omega)\right)$, i.e., $f=f_{0}-\operatorname{div}\left(f_{1}\right)$, with $f_{i} \in L^{p^{\prime}}\left(\Omega_{T}\right), i=0,1$. Then there exists a solution $u$ of $(1.1)$ belonging to $L_{l o c}^{p}\left(0, T ; W_{0}^{1, p}(\Omega)\right) \cap C_{l o c}\left(0, T ; L^{2}(\Omega)\right)$ and satisfying the following estimates

$$
\begin{gathered}
\|u\|_{L^{\infty}\left(\varepsilon, T ; L^{2}(\Omega)\right)} \leq C_{\alpha}\left[\left\|f_{0}\right\|_{L^{p^{\prime}\left(\Omega_{T}\right)}}+\left\|f_{1}\right\|_{L^{p^{\prime}\left(\Omega_{T}\right)}}\right]^{\frac{p^{\prime}}{2}}+B(\varepsilon) \\
\int_{t}^{T} \int_{\Omega}|\nabla u|^{p} \leq c_{0}\left[\left\|f_{0}\right\|_{L^{p^{\prime}\left(\Omega_{T}\right)}}+\left\|f_{1}\right\|_{L^{p^{\prime}}\left(\Omega_{T}\right)}\right]^{p^{\prime}}+\frac{p^{\prime}}{\alpha}[B(t)]^{2} \quad \text { for every } 0<t \leq T
\end{gathered}
$$

where

$$
B(\varepsilon)=\min \left\{\frac{C_{0}}{\varepsilon^{\frac{1}{p-2}}}, C_{1} \frac{\left\|u_{0}\right\|_{M(\Omega)}^{\frac{p}{N(p-2)+p}}}{\varepsilon^{\frac{N}{N(p-2)+p}}}\right\}|\Omega|^{\frac{1}{2}}, \quad \text { if } p>2
$$




$$
B(\varepsilon)=\min \left\{C_{2} \frac{\left\|u_{0}\right\|_{M(\Omega)}}{\varepsilon^{\frac{N}{2}} e^{\sigma \varepsilon}}, C_{1} \frac{\left\|u_{0}\right\|_{M(\Omega)}^{\frac{p}{N(p-2)+p}}}{\varepsilon^{\frac{N}{N(p-2)+p}}}\right\}|\Omega|^{\frac{1}{2}}, \quad \text { if } p=2
$$

where $C_{\alpha}$ and $c_{0}$ as in Theorem 2.5.

In particular, if $p>2$ the universal estimates (2.32) and (2.33) hold true.

Finally, $u$ is obtained as the limit (a.e. in $\Omega_{T}$ ) of the regular solution $u_{n}$ of (4.46).

\section{Preliminary results}

We recall here some known results about $L^{\infty}$-decay estimates proved in [20] that will be essential in the proofs of the outcomes presented here. We observe that in the theorems below only integral estimates are assumed (hence a priori the functions involved could not solve any partial differential equation). Moreover, the proofs of all the results stated in this section make use only of elementary tools (a Lemma on suitable continuous functions proved in [27] and a classical Lemma on nonnegative real numbers often used in the framework of boundedness). We point out that further developments and applications of regularity results proved simply by means of suitable integral inequalities can be found in [19-26].

In detail, let us define

$$
G_{k}(s)=(|s|-k)+\operatorname{sign}(s) .
$$

We have:

Theorem 3.1 (Theorem 2.1 in [20]) Assume that

$$
u \in C\left((0, T) ; L^{r}(\Omega)\right) \cap L^{b}\left(0, T ; L^{q}(\Omega)\right) \cap C\left([0, T) ; L^{r_{0}}(\Omega)\right)
$$

where $\Omega$ is an open set of $\mathbb{R}^{N}, N \geq 1,0<T \leq+\infty$ and

$$
1 \leq r_{0}<r<q \leq+\infty, \quad b_{0}<b<q, \quad b_{0}=\frac{\left(r-r_{0}\right)}{1-\frac{r_{0}}{q}} .
$$

Suppose that $u$ satisfies the following integral estimates for every $k>0$

$$
\begin{aligned}
& \int_{\Omega}\left|G_{k}(u)\right|^{r}\left(t_{2}\right) d x-\int_{\Omega}\left|G_{k}(u)\right|^{r}\left(t_{1}\right) d x \\
& +c_{1} \int_{t_{1}}^{t_{2}}\left\|G_{k}(u)(\tau)\right\|_{L^{q}(\Omega)}^{b} d \tau \leq 0 \quad \text { for every } 0<t_{1}<t_{2}<T \\
& \left\|G_{k}(u)(t)\right\|_{L^{r_{0}(\Omega)}} \leq c_{2}\left\|G_{k}(u)\left(t_{0}\right)\right\|_{L^{r_{0}(\Omega)}} \quad \text { for every } 0 \leq t_{0}<t<T,
\end{aligned}
$$

where $c_{1}$ and $c_{2}$ are positive constants independent of $k$ and

$$
u_{0} \equiv u(x, 0) \in L^{r_{0}}(\Omega) .
$$

Then there exists a positive constant $C_{1}$ (see formula (4.19) in [20]) depending only on $N$, $c_{1}, c_{2}, r, r_{0}, q$ and $b$ such that

$$
\|u(t)\|_{L^{\infty}(\Omega)} \leq C_{1} \frac{\left\|u_{0}\right\|_{L^{r_{0}(\Omega)}}^{h_{0}}}{t^{h_{1}}} \quad \text { for every } t \in(0, T),
$$

where

$$
h_{1}=\frac{1}{b-\left(r-r_{0}\right)-\frac{r_{0} b}{q}}, \quad h_{0}=h_{1}\left(1-\frac{b}{q}\right) r_{0} \text {. }
$$


Moreover, if $\Omega$ has finite measure we have universal bounds if $b>r$ and exponential estimates if $b=r$. More in detail we have the following results.

Theorem 3.2 (Theorem 2.2 in [20]) Let the assumptions of Theorem 3.1 hold true. If $\Omega$ has finite measure and $b>r$ we have the following universal bound

$$
\|u(t)\|_{L^{\infty}(\Omega)} \leq \frac{C_{\sharp}}{t^{h_{2}}} \quad \text { for every } t \in(0, T),
$$

where

$$
h_{2}=h_{1}+\frac{h_{0}}{b-r}=\frac{1}{b-r},
$$

and $C_{\sharp}$, (see formula (4.19) in [20]), is a constant depending only on $r, r_{0}, q, b, c_{1}, c_{2}$ and the measure of $\Omega$.

Theorem 3.3 (Theorem 2.2 in [20]) Let the assumptions of Theorem 3.1 hold true. If $\Omega$ has finite measure and $b=r$ the following exponential bound holds

$$
\|u(t)\|_{L^{\infty}(\Omega)} \leq C_{2} \frac{\left\|u_{0}\right\|_{L^{r_{0}(\Omega)}}}{t^{h_{1}} e^{\sigma t}}, \quad \text { for every } t \in(0, T),
$$

where $C_{2}$ is a positive constant depending only on $N, c_{1}, c_{2}, r, r_{0}, b$ and $q, h_{1}$ is as in (3.8) and

$$
\sigma=\frac{c_{1} \kappa}{4\left(r-r_{0}\right)|\Omega|^{1-\frac{b}{q}}}, \quad \kappa \text { arbitrarily fixed in }\left(0,1-\frac{r_{0}}{r}\right) .
$$

\section{Proofs of results}

In this section, we prove all the results stated in Sect. 2.

\subsection{Proof of Theorem 2.1}

Let $u$ be a solution $u$ of (1.1) obtained as the a.e. limit in $\Omega_{T}$ of the solutions $u_{n} \in L^{\infty}\left(\Omega_{T}\right) \cap$ $C\left([0, T] ; L^{2}(\Omega)\right) \cap L^{p}\left(0, T ; W_{0}^{1, p}(\Omega)\right)$ of the following approximating problems

$$
\begin{cases}\left(u_{n}\right)_{t}-\operatorname{div}\left(a\left(x, t, \nabla u_{n}\right)\right)=f_{n}(x, t) & \text { in } \Omega_{T}, \\ u_{n}=0 & \text { on } \Gamma, \\ u_{n}(x, 0)=u_{0, n}(x) & \text { on } \Omega,\end{cases}
$$

where the data $u_{0, n}(x) \in L^{\infty}(\Omega)$ and $f_{n}(x, t) \in L^{\infty}\left(\Omega_{T}\right)$ satisfy

$$
\begin{aligned}
& u_{0, n}(x) \rightarrow u_{0} \quad \text { in } L^{1}(\Omega), \\
& f_{n}(x, t) \rightarrow f(x, t) \quad \text { in } L^{1}\left(\Omega_{T}\right),
\end{aligned}
$$

(see [7]). Notice that being $u_{n}$ in $C\left([0, T] ; L^{2}(\Omega)\right)$ we can choose in (2.2) also test functions $\varphi$ not satisfying $\varphi(T)=0$ and in these last cases in the left-hand side of (2.2) will appear also the integral on $\Omega$ of $u_{n}(T) \varphi(T)$. Indeed, it results

$$
u \in L^{q}\left(0, T ; W_{0}^{1, q}(\Omega)\right) \quad \text { for every } \quad 1 \leq q<\frac{p(N+1)-N}{N+1}
$$

(see again [7]) and

$$
u \in C\left([0, T] ; L^{1}(\Omega)\right),
$$


(see [28]). Analogously, let $v \in C\left([0, T] ; L^{1}(\Omega)\right) \cap L^{q}\left(0, T ; W_{0}^{1, q}(\Omega)\right)$ be a solution of (2.3) obtained as the a.e. limit in $\Omega_{T}$ of the sequence $v_{n} \in L^{\infty}\left(\Omega_{T}\right) \cap C\left([0, T] ; L^{2}(\Omega)\right) \cap$ $L^{p}\left(0, T ; W_{0}^{1, p}(\Omega)\right)$ solutions of the following approximating problems

$$
\begin{cases}\left(v_{n}\right)_{t}-\operatorname{div}\left(a\left(x, t, \nabla v_{n}\right)\right)=f_{n}(x, t) & \text { in } \Omega_{T} \\ v_{n}=0 & \text { on } \Gamma \\ v_{n}(x, 0)=v_{0, n}(x) & \text { on } \Omega\end{cases}
$$

where $v_{0, n}(x) \in L^{\infty}(\Omega)$ satisfies

$$
v_{0, n}(x) \rightarrow v_{0} \quad \text { in } L^{1}(\Omega),
$$

and with $f_{n}$ the same approximating sequence defined in (4.3). Let $k$ a positive constant arbitrarily fixed and $G_{k}(s)$ the real function defined in (3.1). Choose $G_{k}\left(u_{n}-v_{n}\right)$ as test function in (4.1) and in (4.4). Notice that these choices, together with the following ones can be made rigorous by means of the Steklov averaging process. Subtracting the equations obtained in this way we get for every $0<t_{1}<t_{2}<T$

$$
\begin{aligned}
& \frac{1}{2} \int_{\Omega}\left|G_{k}\left(u_{n}-v_{n}\right)\left(t_{2}\right)\right|^{2}-\frac{1}{2} \int_{\Omega}\left|G_{k}\left(u_{n}-v_{n}\right)\left(t_{1}\right)\right|^{2} \\
& \quad+\int_{t_{1}}^{t_{2}} \int_{\Omega}\left[a\left(x, t, \nabla u_{n}\right)-a\left(x, t, \nabla v_{n}\right)\right] \nabla G_{k}\left(u_{n}-v_{n}\right)=0 .
\end{aligned}
$$

By (1.3) the previous estimate implies

$$
\begin{aligned}
& \frac{1}{2} \int_{\Omega}\left|G_{k}\left(u_{n}-v_{n}\right)\left(t_{2}\right)\right|^{2}-\frac{1}{2} \int_{\Omega}\left|G_{k}\left(u_{n}-v_{n}\right)\left(t_{1}\right)\right|^{2} \\
& \quad+\alpha \int_{t_{1}}^{t_{2}} \int_{\Omega}\left|\nabla G_{k}\left(u_{n}-v_{n}\right)\right|^{p} \leq 0,
\end{aligned}
$$

and hence, thanks to the Sobolev inequality ${ }^{4}$ we obtain

$$
\int_{\Omega}\left|G_{k}\left(u_{n}-v_{n}\right)\left(t_{2}\right)\right|^{2}-\int_{\Omega}\left|G_{k}\left(u_{n}-v_{n}\right)\left(t_{1}\right)\right|^{2}+2 \alpha c_{s} \int_{t_{1}}^{t_{2}}\left\|G_{k}\left(u_{n}-v_{n}\right)\right\|_{L^{p^{*}}(\Omega)}^{p} \leq 0 .
$$

Notice that the previous inequality is equivalent to require that the assumption (3.4) is satisfied by $u_{n}-v_{n}$ with $c_{1}=2 \alpha c_{s}, r=2, b=p$ and $q=p^{*}$. Observe also that the previous choice of the coefficients $\mathrm{r}, \mathrm{b}$ and $\mathrm{q}$ satisfies the condition (3.3) with $r_{0}=1$. Moreover, it results $b>r$. Hence to apply Theorem 3.2, we need to prove that also (3.5) is verified. To this aim, let $\delta>1$ arbitrarily fixed and take as test function $\varphi=\left\{1-\frac{1}{\left[1+\left|G_{k}\left(u_{n}-v_{n}\right)\right|\right]^{\delta}}\right\} \operatorname{sign}\left(u_{n}-v_{n}\right)$ in both the problems (4.1) and (4.4); subtracting the equations obtained in this way we get for every $0 \leq t_{0}<t<T$

4 The Sobolev inequality: there exists a constant $c_{S}$ depending only on $N$ and p $(N>2)$ such that

$$
c_{S}\left(\int_{\Omega}|v|^{p^{*}} \mathrm{~d} x\right)^{\frac{p}{p^{*}}} \leq \int_{\Omega}|\nabla v|^{p} \mathrm{~d} x \quad \text { for every } v \in W_{0}^{1, p}(\Omega), \quad p^{*}=\frac{p N}{N-p} .
$$




$$
\begin{aligned}
& \int_{\Omega}\left|G_{k}\left(u_{n}-v_{n}\right)\right|(t)+\frac{1}{1-\delta} \int_{\Omega}\left\{1-\frac{1}{\left[1+\left|G_{k}\left(u_{n}-v_{n}\right)\right|(t)\right]^{\delta-1}}\right\} \\
& \quad+\delta \int_{t_{0}}^{t} \int_{\Omega}\left[a\left(x, t, \nabla u_{n}\right)-a\left(x, t, \nabla v_{n}\right)\right] \nabla G_{k}\left(u_{n}-v_{n}\right) \frac{1}{\left[1+\left|G_{k}\left(u_{n}-v_{n}\right)\right|(t)\right]^{\delta+1}} \\
& \quad \leq \int_{\Omega}\left|G_{k}\left(u_{n}-v_{n}\right)\right|\left(t_{0}\right)+\frac{1}{1-\delta} \int_{\Omega}\left\{1-\frac{1}{\left[1+\left|G_{k}\left(u_{n}-v_{n}\right)\right|\left(t_{0}\right)\right]^{\delta-1}}\right\},
\end{aligned}
$$

from which, thanks to assumption (1.3), we obtain for every $0 \leq t_{0}<t<T$ and $\delta>1$

$$
\begin{aligned}
& \int_{\Omega}\left|G_{k}\left(u_{n}-v_{n}\right)\right|(t) \leq \int_{\Omega}\left|G_{k}\left(u_{n}-v_{n}\right)\right|\left(t_{0}\right)+\frac{1}{\delta-1} \int_{\Omega} \frac{1}{\left[1+\left|G_{k}\left(u_{n}-v_{n}\right)\right|(t)\right]^{\delta-1}} \\
& \quad \leq \int_{\Omega}\left|G_{k}\left(u_{n}-v_{n}\right)\right|\left(t_{0}\right)+\frac{|\Omega|}{\delta-1} .
\end{aligned}
$$

Hence (letting $\delta \rightarrow+\infty$ ) we deduce

$$
\int_{\Omega}\left|G_{k}\left(u_{n}-v_{n}\right)\right|(t) \leq \int_{\Omega}\left|G_{k}\left(u_{n}-v_{n}\right)\right|\left(t_{0}\right),
$$

i.e., (3.5) is satisfied. Thus we can apply Theorem 3.2 obtaining the following universal estimate

$$
\left\|u_{n}(t)-v_{n}(t)\right\|_{L^{\infty}(\Omega)} \leq \frac{C_{\sharp}}{t^{\frac{1}{p-2}}} \quad \text { for every } t \in(0, T),
$$

where the positive constant $C_{\sharp}$ depends only on $N, p$ and $\alpha$. Noticing that $u_{n}-v_{n}$ converges a.e. to $u-v$ in $\Omega_{T}$ by the previous estimate we deduce

$$
\|u(t)-v(t)\|_{L^{\infty}(\Omega)} \leq \frac{C_{0}}{t^{\frac{1}{p-2}}} \quad \text { for almost every } t \in(0, T),
$$

where $C_{0}=C_{\sharp}$. We observe that also the assumptions of Theorem 3.1 are satisfied and consequently also the following estimate holds true

$$
\left\|u_{n}(t)-v_{n}(t)\right\|_{L^{\infty}(\Omega)} \leq C_{1} \frac{\left\|u_{0, n}-v_{0, n}\right\|_{L^{1}(\Omega)}^{\frac{p}{N(p-2)+p}}}{t^{\frac{N}{N(p-2)+p}}} \quad \text { for every } t \in(0, T),
$$

where $C_{1}$ is a positive constant depending only on $N, p$ and $\alpha$. Hence by (4.12) we obtain

$$
\|u(t)-v(t)\|_{L^{\infty}(\Omega)} \leq C_{1} \frac{\left\|u_{0}-v_{0}\right\|_{L^{1}(\Omega)}^{\frac{p}{N(p-2)+p}}}{t^{\frac{N}{N(p-2)+p}}} \quad \text { for almost every } t \in(0, T) .
$$

By (4.11) and (4.13) it follows that estimate (2.5) holds true.

Remark 4.1 We notice that if in (4.1) we change the approximation of the initial datum $u_{0}$, i.e., if we consider another approximation, as for example

$$
\begin{cases}\left(z_{n}\right)_{t}-\operatorname{div}\left(a\left(x, t, \nabla z_{n}\right)\right)=f_{n}(x, t) & \text { in } \Omega_{T}, \\ z_{n}=0 & \text { on } \Gamma \\ z_{n}(x, 0)=z_{0, n}(x) & \text { on } \Omega\end{cases}
$$

where the data $z_{0, n}(x) \in L^{\infty}(\Omega)$ satisfy

$$
z_{0, n}(x) \rightarrow u_{0} \quad \text { in } L^{1}(\Omega),
$$


and $f_{n}(x, t) \in L^{\infty}\left(\Omega_{T}\right)$ are as in (4.3) then by estimate (4.12) it follows that

$$
\left\|u_{n}(t)-z_{n}(t)\right\|_{L^{\infty}(\Omega)} \leq C_{1} \frac{\left\|u_{0, n}-z_{0, n}\right\|_{L^{1}(\Omega)}^{\frac{p}{N(p-2)+p}}}{t^{\frac{N}{N(p-2)+p}}} \quad \text { for every } t \in(0, T),
$$

and hence both these approximations will converge to the same solution $u$ of (1.1). In other words, changing the approximation of the initial datum, the solution $u$ that we obtain as a limit does not change. The uniqueness of the solutions obtained by approximation is a known result but here we obtain a different proof that make use of the "continuity estimate" (4.12) (or, equivalently, of (4.13)).

\subsection{Proof of Theorem 2.2}

Let $T_{0}>0$ arbitrarily fixed. By Theorem 2.1 there exists a solution $U_{0} \in C\left(\left[0, T_{0}\right] ; L^{1}(\Omega)\right)$ of

$$
\begin{cases}\left(U_{0}\right)_{t}-\operatorname{div}\left(a\left(x, t, \nabla U_{0}\right)\right)=f(x, t) & \text { in } \Omega_{T_{0}}, \\ U_{0}=0 & \text { on } \partial \Omega \times\left(0, T_{0}\right), \\ U_{0}(x, 0)=u_{0}(x) & \text { on } \Omega\end{cases}
$$

obtained as a.e. limit in $\Omega \times\left(0, T_{0}\right)$ of regular solutions $u_{n} \in L^{\infty}\left(\Omega_{T_{0}}\right) \cap C\left(\left[0, T_{0}\right] ; L^{2}(\Omega)\right) \cap$ $L^{p}\left(0, T_{0} ; W_{0}^{1, p}(\Omega)\right)$ of the following approximating problems

$$
\begin{cases}\left(u_{n}\right)_{t}-\operatorname{div}\left(a\left(x, t, \nabla u_{n}\right)\right)=f_{n}^{0}(x, t) & \text { in } \Omega_{T_{0}}, \\ u_{n}=0 & \text { on } \partial \Omega \times\left(0, T_{0}\right), \\ u_{n}(x, 0)=u_{0, n}(x) & \text { on } \Omega\end{cases}
$$

where the data $u_{0, n}(x) \in L^{\infty}(\Omega)$ are as in (4.2) and $f_{n}^{0}(x, t) \in L^{\infty}\left(\Omega_{T_{0}}\right)$ satisfy

$$
f_{n}^{0}(x, t) \rightarrow f(x, t) \quad \text { in } L^{1}\left(\Omega_{T_{0}}\right) .
$$

With a similar construction and again by Theorem 2.1, we obtain a solution $U_{1}$ belonging to $C\left(\left[T_{0}, 2 T_{0}\right] ; L^{1}(\Omega)\right)$ of

$$
\begin{cases}\left(U_{1}\right)_{t}-\operatorname{div}\left(a\left(x, t, \nabla U_{1}\right)\right)=f(x, t) & \text { in } \Omega \times\left(T_{0}, 2 T_{0}\right), \\ U_{1}=0 & \text { on } \partial \Omega \times\left(T_{0}, 2 T_{0}\right), \\ U_{1}\left(x, T_{0}\right)=U_{0}\left(T_{0}\right) & \text { on } \Omega,\end{cases}
$$

obtained as a.e. limit in $\Omega \times\left(T_{0}, 2 T_{0}\right)$ of regular solutions (that for sake of notation we denote again $\left.u_{n}\right)$ problems $u_{n} \in L^{\infty}\left(\Omega \times\left(T_{0}, 2 T_{0}\right)\right) \cap C\left(\left[T_{0}, 2 T_{0}\right] ; L^{2}(\Omega)\right) \cap L^{p}\left(T_{0}, 2 T_{0} ; W_{0}^{1, p}(\Omega)\right)$ of the following approximating problems

$$
\begin{cases}\left(u_{n}\right)_{t}-\operatorname{div}\left(a\left(x, t, \nabla u_{n}\right)\right)=f_{n}^{1}(x, t) & \text { in } \Omega \times\left(T_{0}, 2 T_{0}\right), \\ u_{n}=0 & \text { on } \partial \Omega \times\left(T_{0}, 2 T_{0}\right), \\ u_{n}(x, 0)=u_{1, n}(x) & \text { on } \Omega,\end{cases}
$$

where the data $u_{1, n}(x) \in L^{\infty}(\Omega)$ are regular approximations of $U_{0}\left(T_{0}\right)$ (hence satisfying similar converging properties in (4.2)) and $f_{n}^{1}(x, t) \in L^{\infty}\left(\Omega \times\left(T_{0}, 2 T_{0}\right)\right)$ satisfy

$$
f_{n}^{1}(x, t) \rightarrow f(x, t) \quad \text { in } L^{1}\left(\Omega \times\left(T_{0}, 2 T_{0}\right)\right) .
$$


Iterating this process, we construct a sequence of solutions $U_{i}$ in $C\left(\left[i T_{0},(i+1) T_{0}\right] ; L^{1}(\Omega)\right)$ $(i \in \mathbf{N})$ of

$$
\begin{cases}\left(U_{i}\right)_{t}-\operatorname{div}\left(a\left(x, t, \nabla U_{i}\right)\right)=f(x, t) & \text { in } \Omega \times\left(i T_{0},(i+1) T_{0}\right), \\ U_{i}=0 & \text { on } 2 \Omega \times\left(i T_{0},(i+1) T_{0}\right), \\ U_{i}\left(x, i T_{0}\right)=U_{i-1}\left(i T_{0}\right) & \text { on } \Omega,\end{cases}
$$

obtained by approximation. We define $u=U_{i}$ in $\Omega \times\left[i T_{0},(i+1) T_{0}\right]$, for every $i \in \mathbf{N}$. By construction it follows that $u$ is a global solution (according to Definition 2.2). In a similar way, using the same approximation of $\mathrm{f}$ defined above (i.e., $f_{n}(x, t)=f_{n}^{i}(x, t)$ in $\Omega \times\left(i T_{0},(i+1) T_{0}\right)$ for every $\left.i \in \mathbf{N}\right)$ we construct a global solution $v$ of (2.3). Now the assert follows by Theorem 2.1 .

\subsection{Proof of Corollary 2.1}

Let $\mathrm{w}$ be the solution of the stationary problem (2.14) obtained as the limit in $L^{1}(\Omega)$ of the solutions $w_{n} \in W_{0}^{1, p}(\Omega) \cap L^{\infty}(\Omega)$ of (2.15) where $f_{n} \in L^{\infty}(\Omega)$ satisfy (2.16). Passing eventually to subsequences, we can assume that $w_{n}$ converges a.e. in $\Omega$ to w. We observe that $w(x, t) \equiv w(x)$ is also a global solution (according to Definition 2.2) of the following problem

$$
\begin{cases}w_{t}-\operatorname{div}(a(x, \nabla w))=f(x) & \text { in } \Omega_{T} \\ w=0 & \text { on } \Gamma \\ w(x, 0)=w(x) & \text { on } \Omega\end{cases}
$$

Moreover, the approximating functions $w_{n}(x) \equiv w_{n}(x, t)$ are global solutions too but of the following problems

$$
\begin{cases}\left(w_{n}\right)_{t}-\operatorname{div}\left(a\left(x, \nabla w_{n}\right)\right)=f_{n}(x) & \text { in } \Omega_{T} \\ w_{n}=0 & \text { on } \Gamma \\ w_{n}(x, 0)=w_{n}(x) & \text { on } \Omega\end{cases}
$$

Let $u$ be the global solution of (1.1) constructed in the proof of Theorem 2.2, i.e., $u$ is the a.e. limit in $\Omega_{T}$ (for every arbitrarily fixed $T>0$ ) of the solutions $u_{n} \in L^{\infty}\left(\Omega_{T}\right) \cap$ $C\left([0, T] ; L^{2}(\Omega)\right) \cap L^{p}\left(0, T ; W_{0}^{1, p}(\Omega)\right)$ of the following approximating problems

$$
\begin{cases}\left(u_{n}\right)_{t}-\operatorname{div}\left(a\left(x, \nabla u_{n}\right)\right)=f_{n}(x) & \text { in } \Omega_{T} \\ u_{n}=0 & \text { on } \Gamma \\ u_{n}(x, 0)=u_{0, n}(x) & \text { on } \Omega\end{cases}
$$

where the data $u_{0, n}(x) \in L^{\infty}(\Omega)$ satisfy (4.2) and $f_{n}$ is the same sequence chosen above in (4.23). Now the assert follows applying Theorem 2.1 .

\subsection{Proof of Theorem 2.3}

Let $u^{0}$ be (as in the statement of the theorem) the solution of (1.14) obtained as limit of the approximating solutions $u_{n}^{0}$ of (2.17). Construct $u$ as the solution in $C\left([0, T] ; L^{1}(\Omega)\right)$ obtained as the limit (a.e. in $\Omega_{T}$ ) of the solutions of the approximating problems (4.1) where $f_{n}$ is the same approximating sequence of $f$ chosen in (2.17). Hence, by Theorem 2.1 (applied choosing $v=u^{0}$ ) we deduce that (2.5) holds true, i.e., 


$$
\left\|u(t)-u^{0}(t)\right\|_{L^{\infty}(\Omega)} \leq \min \left\{\frac{C_{0}}{t^{\frac{1}{p-2}}}, C_{1} \frac{\left\|u_{0}\right\|_{L^{1}(\Omega)}^{\frac{p}{N(p-2)+p}}}{t^{\frac{N}{N(p-2)+p}}}\right\} \quad \text { for almost every } t \in(0, T)
$$

from which it follows that for almost every $(x, t) \in \Omega_{T}$

$$
|u(x, t)| \leq\left|u^{0}(x, t)\right|+\min \left\{\frac{C_{0}}{t^{\frac{1}{p-2}}}, C_{1} \frac{\left\|u_{0}\right\|_{L^{1}(\Omega)}^{\frac{p}{N(p-2)+p}}}{t^{\frac{N}{N(p-2)+p}}}\right\} .
$$

Now the claims (2.19) and (2.20) gather by the previous estimate.

\subsection{Proof of Corollary 2.2}

The assertions follow by the results in Theorem 2.3 together with the regularity results (applied to $u^{0}$ ) in $[6,12,18]$.

\subsection{Proof of Theorem 2.4}

By Theorem 2.3 there exists a solution $u$ of (1.1) which belongs to $C\left([0, T] ; L^{1}(\Omega)\right)$ and satisfies (2.19) and (2.20). Moreover, $u$ is obtained as the limit (a.e. in $\Omega_{T}$ ) of the approximating solution $u_{n} \in L^{\infty}\left(\Omega_{T}\right) \cap C\left([0, T] ; L^{2}(\Omega)\right) \cap L^{p}\left(0, T ; W_{0}^{1, p}(\Omega)\right)$ of (4.1). It is not restrictive to assume that the approximating sequence $f_{n}$ satisfy

$$
\left\|f_{n}\right\|_{L^{1}\left(\Omega_{T}\right)} \leq\|f\|_{L^{1}\left(\Omega_{T}\right)} .
$$

Since assumption (2.22) implies that $u^{0}$ belongs to $L^{\infty}\left(\Omega_{T}\right)$, it follows that for every $\varepsilon \in$ $(0, T)$ arbitrarily fixed, $u$ belongs to $L^{\infty}\left(\varepsilon, T ; L^{\infty}(\Omega)\right)$ and satisfies

$$
\|u\|_{L^{\infty}\left(\varepsilon, T ; L^{\infty}(\Omega)\right)} \leq\left\|u^{0}\right\|_{L^{\infty}\left(\varepsilon, T ; L^{\infty}(\Omega)\right)}+\min \left\{\frac{C_{0}}{\varepsilon^{\frac{1}{p-2}}}, C_{1} \frac{\left\|u_{0}\right\|_{L^{1}(\Omega)}^{\frac{p}{N(p-2)+p}}}{\varepsilon^{\frac{N}{N(p-2)+p}}}\right\} .
$$

By the previous estimate and recalling that $u^{0}$ satisfies the classical $L^{\infty}$-estimate

$$
\left\|u^{0}\right\|_{L^{\infty}\left(\Omega_{T}\right)} \leq c_{0}\|f\|_{L^{m}\left(0, T ; L^{s}(\Omega)\right)},
$$

(where $c_{0}$ depends only on $T, N, \alpha, p$ and $|\Omega|$ ) we conclude that estimate (2.24) holds true. We show now that also the gradient of $u$ has an immediate improvement in regularity and satisfies estimate (2.25).

To this aim, take $u_{n}$ as test function in (4.1). We deduce that for every $0<t \leq T$

$$
\frac{1}{2} \int_{\Omega}\left|u_{n}(T)\right|^{2}+\alpha \int_{t}^{T} \int_{\Omega}\left|\nabla u_{n}\right|^{p} \leq \frac{1}{2} \int_{\Omega}\left|u_{n}(t)\right|^{2}+\int_{t}^{T} \int_{\Omega} f_{n} u_{n} .
$$

Notice that we can estimate the right-hand side of the previous inequality as follows. Thanks to (2.24) we have

$$
\frac{1}{2} \int_{\Omega}\left|u_{n}(t)\right|^{2} \leq \frac{|\Omega|}{2}[A(t)]^{2},
$$


where $A(t)$ is as in (2.24). Moreover, it results (thanks to (4.26))

$$
\int_{t}^{T} \int_{\Omega} f_{n} u_{n} \leq\left\|u_{n}\right\|_{L^{\infty}(\Omega \times(t, T))}\|f\|_{L^{1}\left(\Omega_{T}\right)} .
$$

Hence, using again estimate (2.24) we deduce

$$
\left\|u_{n}\right\|_{L^{\infty}(\Omega \times(t, T))} \leq \sup _{\tau \in(t, T)}\left\|u_{n}(\tau)\right\|_{L^{\infty}(\Omega)} \leq \sup _{\tau \in(t, T)} A(\tau)=A(t)
$$

Thus by the previous inequalities we obtain

$$
\int_{t}^{T} \int_{\Omega} f_{n} u_{n} \leq A(t)\|f\|_{L^{1}\left(\Omega_{T}\right)} .
$$

By (4.28)-(4.31) we conclude that

$$
\int_{t}^{T} \int_{\Omega}\left|\nabla u_{n}\right|^{p} \leq \frac{1}{\alpha} A(t)\left(\frac{|\Omega|}{2} A(t)+\|f\|_{L^{1}\left(\Omega_{T}\right)}\right),
$$

which implies the assertion (2.25).

We observe that from what proved above it follows that $u$ belongs to $C\left((0, T) ; L^{2}(\Omega)\right)$. As a matter of fact, the sequence $u_{n}$ is a Cauchy sequence in $C\left([0, T] ; L^{1}(\Omega)\right)$ and by (4.30) it is also equi-bounded in $[\varepsilon, T] \times \Omega$ (by $A(\varepsilon)$ ) for every $\varepsilon>0$ arbitrarily fixed. Hence $u_{n}$ is also a Cauchy sequence in $C\left([\varepsilon, T] ; L^{2}(\Omega)\right)$ for every $\varepsilon>0$ arbitrarily fixed and consequently $u$ belongs to $C\left([\varepsilon, T] ; L^{2}(\Omega)\right)$ (for every $\varepsilon>0$ arbitrarily fixed), i.e., $u$ is in $C\left((0, T) ; L^{2}(\Omega)\right.$ ).

Now, observing that $u$ belongs to $L^{\infty}\left((\varepsilon, T) ; L^{\infty}(\Omega)\right) \cap C\left((0, T) ; L^{2}(\Omega)\right) \cap L^{p}((\varepsilon, T)$; $W_{0}^{1, p}(\Omega)$ ) (for every $\varepsilon \in(0, T)$ arbitrarily fixed) the Hoelder continuity of $u$ in $\bar{\Omega} \times(\varepsilon, T)$ follows by the result in [13] (see also [12]).

\subsection{Proof of Theorem 2.5}

Since the datum $\mathrm{f}$ is in the dual space $L^{p^{\prime}}\left(0, T ; W^{-1, p^{\prime}}(\Omega)\right)$, we can construct a solution $u$ of (1.1) as the a.e. limit in $\Omega_{T}$ of the solutions $u_{n} \in C\left([0, T] ; L^{2}(\Omega)\right) \cap L^{p}\left(0, T ; W_{0}^{1, p}(\Omega)\right)$ of the approximating problems

$$
\begin{cases}\left(u_{n}\right)_{t}-\operatorname{div}\left(a\left(x, \nabla u_{n}\right)\right)=f & \text { in } \Omega_{T}, \\ u_{n}=0 & \text { on } \Gamma, \\ u_{n}(x, 0)=u_{0, n}(x) & \text { on } \Omega,\end{cases}
$$

where the sequence $u_{0, n}$ satisfies (4.2) and, differently from the previous approximation problems (4.1) above, now it is not necessary to approximate also the datum $\mathrm{f}$ with more regular data $f_{n}$. Hence now, differently from above, generally $u_{n}$ doesn't belong to $L^{\infty}\left(\Omega_{T}\right)$. Anyway, the regularity of the approximating sequence $u_{n}$ is enough to conclude (doing the same calculations in the proofs of Theorems 2.1 and 2.3 ) that also now (2.19) and (2.20) hold true with $u^{0}$ the unique solution of (1.14) belonging to $C\left([0, T] ; L^{2}(\Omega)\right) \cap$ $L^{p}\left(0, T ; W_{0}^{1, p}(\Omega)\right)$.

Thus, being $u^{0}$ in $L^{\infty}\left(0, T ; L^{2}(\Omega)\right)$, by (2.19) and (2.20) it follows that $\mathrm{u}$ belongs to $L^{\infty}\left(\varepsilon, T ; L^{2}(\Omega)\right)$ (for every positive $\varepsilon$ ) and verifies

$$
\|u\|_{L^{\infty}\left(\varepsilon, T ; L^{2}(\Omega)\right)} \leq\left\|u^{0}\right\|_{L^{\infty}\left(\varepsilon, T ; L^{2}(\Omega)\right)}+\min \left\{\frac{C_{0}}{\varepsilon^{\frac{1}{p-2}}}, C_{1} \frac{\left\|u_{0}\right\|_{L^{1}(\Omega)}^{\frac{p}{N(p-2)+p}}}{\varepsilon^{\frac{N}{N(p-2)+p}}}\right\}|\Omega|^{\frac{1}{2}},
$$


where $C_{0}$ and $C_{1}$ are as in (2.5). Hence, to obtain an estimate for $u$ by the previous inequality we need to estimate the norm $\left\|u^{0}\right\|_{L^{\infty}\left(\varepsilon, T ; L^{2}(\Omega)\right)}$. To this aim taking $u^{0}$ as test function in (1.14) and using (1.5) we deduce for every $0<t \leq T$

$$
\begin{aligned}
& \frac{1}{2} \int_{\Omega}\left|u^{0}(t)\right|^{2}+\alpha \int_{0}^{t} \int_{\Omega}\left|\nabla u^{0}\right|^{p} \\
& \leq \int_{0}^{t} \int_{\Omega} f_{0} u^{0}+\int_{0}^{t} \int_{\Omega} f_{1} \nabla u^{0} .
\end{aligned}
$$

We estimate now the right-hand side of the previous inequality. We have (using Young and Poincaré ${ }^{5}$ inequalities)

$$
\begin{aligned}
& \int_{0}^{t} \int_{\Omega} f_{0} u^{0}+\int_{0}^{t} \int_{\Omega} f_{1} \nabla u^{0} \leq\left\|f_{0}\right\|_{L^{p^{\prime}\left(\Omega_{T}\right)}}\left\|u^{0}\right\|_{L^{p}(\Omega \times(t, T))}+\left\|f_{1}\right\|_{L^{p^{\prime}\left(\Omega_{T}\right)}}\left\|\nabla u^{0}\right\|_{L^{p}(\Omega \times(t, T))} \\
& \leq\left[\left\|f_{0}\right\|_{L^{p^{\prime}}\left(\Omega_{T}\right)} c_{P}+\left\|f_{1}\right\|_{L^{p^{\prime}\left(\Omega_{T}\right)}}\right]\left\|\nabla u^{0}\right\|_{L^{p}(\Omega \times(t, T))} \\
& \leq \frac{\alpha}{p} \int_{t}^{T} \int_{\Omega}\left|\nabla u^{0}\right|^{p}+\frac{1}{p^{\prime} \alpha^{\frac{1}{p-1}}}\left[\left\|f_{0}\right\|_{L^{p^{\prime}\left(\Omega_{T}\right)}} c_{P}+\left\|f_{1}\right\|_{L^{p^{\prime}\left(\Omega_{T}\right)}}\right]^{p^{\prime}} \\
& \leq \frac{\alpha}{p} \int_{t}^{T} \int_{\Omega}\left|\nabla u^{0}\right|^{p}+\frac{1}{p^{\prime} \alpha^{\frac{1}{p-1}}}\left(\max \left\{1, c_{P}\right\}\right)^{p^{\prime}}\left[\left\|f_{0}\right\|_{L^{p^{\prime}\left(\Omega_{T}\right)}}+\left\|f_{1}\right\|_{L^{p^{\prime}}\left(\Omega_{T}\right)}\right]^{p^{\prime}}, \quad \text { (4.37) }
\end{aligned}
$$

where $c_{P}$ is the Poincaré constant defined in (4.36). By the previous two estimates, we deduce that for every $0<t \leq T$

$$
\begin{aligned}
& \frac{1}{2} \int_{\Omega}\left|u^{0}(t)\right|^{2}+\frac{\alpha}{p^{\prime}} \int_{0}^{t} \int_{\Omega}\left|\nabla u^{0}\right|^{p} \\
& \leq \frac{1}{p^{\prime} \alpha^{\frac{1}{p-1}}}\left(\max \left\{1, c_{P}\right\}\right)^{p^{\prime}}\left[\left\|f_{0}\right\|_{L^{p^{\prime}\left(\Omega_{T}\right)}}+\left\|f_{1}\right\|_{L^{p^{\prime}}\left(\Omega_{T}\right)}\right]^{p^{\prime}},
\end{aligned}
$$

which implies

$$
\left\|u^{0}\right\|_{L^{\infty}\left(0, T ; L^{2}(\Omega)\right)} \leq C_{\alpha}\left[\left\|f_{0}\right\|_{L^{p^{\prime}}\left(\Omega_{T}\right)}+\left\|f_{1}\right\|_{L^{p^{\prime}}\left(\Omega_{T}\right)}\right]^{\frac{p^{\prime}}{2}}
$$

where $C_{\alpha}=\left[\frac{2}{p^{\prime} \alpha^{\frac{1}{p-1}}}\left(\max \left\{1, c_{P}\right\}\right)^{p^{\prime}}\right]^{\frac{1}{2}}$. By (4.39) and (4.34) it follows

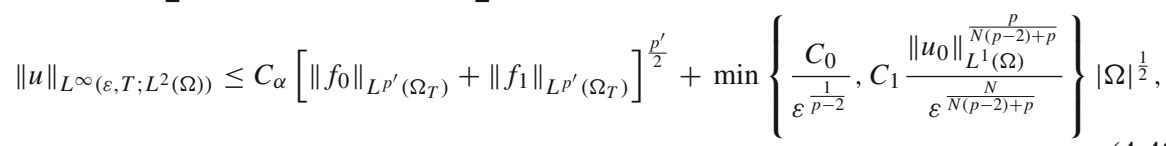

and hence estimate (2.30) is proved.

We show now that also the gradient of $u$ has an immediately improvement in regularity. To this aim, choose $u_{n}$ as test function in (4.33). We obtain

5 The Poincaré inequality: there exists a constant $c_{P}$ depending only on $\Omega, \mathrm{N}$ and $\mathrm{p}$ such that

$$
\|v\|_{L^{p}(\Omega)} \leq c_{P}\|\nabla v\|_{L^{p}(\Omega)} \quad \text { for every } v \in W_{0}^{1, p}(\Omega) .
$$




$$
\begin{aligned}
& \frac{1}{2} \int_{\Omega}\left|u_{n}(T)\right|^{2}+\alpha \int_{t}^{T} \int_{\Omega}\left|\nabla u_{n}\right|^{p} \\
& \quad \leq \frac{1}{2} \int_{\Omega}\left|u_{n}(t)\right|^{2}+\int_{t}^{T} \int_{\Omega} f_{0} u_{n}+\int_{t}^{T} \int_{\Omega} f_{1} \nabla u_{n}, \quad \text { for every } 0<t \leq T .
\end{aligned}
$$

Proceeding as in the proof of (4.40) we deduce that

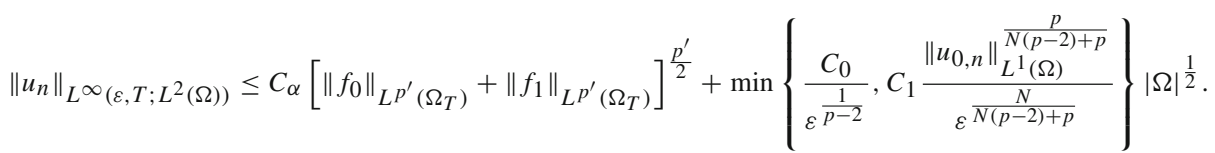

Moreover, by reasoning exactly as in (4.37) we get

$$
\begin{aligned}
& \int_{t}^{T} \int_{\Omega} f_{0} u_{n}+\int_{t}^{T} \int_{\Omega} f_{1} \nabla u_{n} \\
& \leq \frac{\alpha}{p} \int_{t}^{T} \int_{\Omega}\left|\nabla u_{n}\right|^{p}+\frac{1}{p^{\prime} \alpha^{\frac{1}{p-1}}}\left(\max \left\{1, c_{P}\right\}\right)^{p^{\prime}}\left[\left\|f_{0}\right\|_{L^{p^{\prime}}\left(\Omega_{T}\right)}+\left\|f_{1}\right\|_{L^{p^{\prime}}\left(\Omega_{T}\right)}\right]^{p^{\prime}} .
\end{aligned}
$$

By (4.41)-(4.43) it follows

$$
\begin{aligned}
& \frac{\alpha}{p^{\prime}} \int_{t}^{T} \int_{\Omega}\left|\nabla u_{n}\right|^{p} \\
& \leq \frac{1}{2}\left[C_{\alpha}\left[\left\|f_{0}\right\|_{L^{p^{\prime}}\left(\Omega_{T}\right)}+\left\|f_{1}\right\|_{L^{p^{\prime}}\left(\Omega_{T}\right)}\right]^{\frac{p^{\prime}}{2}}+\min \left\{\frac{C_{0}}{t^{\frac{1}{p-2}}}, C_{1} \frac{\left\|u_{0, n}\right\|_{L^{1}(\Omega)}^{\frac{p}{N(p-2)+p}}}{t^{\frac{N}{N(p-2)+p}}}\right\}|\Omega|^{\frac{1}{2}}\right]^{2} \\
& +\frac{1}{p^{\prime} \alpha^{\frac{1}{p-1}}}\left(\max \left\{1, c_{P}\right\}\right)^{p^{\prime}}\left[\left\|f_{0}\right\|_{L^{p^{\prime}}\left(\Omega_{T}\right)}+\left\|f_{1}\right\|_{L^{p^{\prime}}\left(\Omega_{T}\right)}\right]^{p^{\prime}}, \quad \text { for every } 0<t \leq T,
\end{aligned}
$$

that is

$$
\begin{aligned}
& \int_{t}^{T} \int_{\Omega}\left|\nabla u_{n}\right|^{p} \\
& \leq \frac{p^{\prime}}{\alpha} C_{\alpha}^{2}\left[\left\|f_{0}\right\|_{L^{p^{\prime}}\left(\Omega_{T}\right)}+\left\|f_{1}\right\|_{L^{p^{\prime}}\left(\Omega_{T}\right)}\right]^{p^{\prime}}+\frac{p^{\prime}}{\alpha} \min \left\{\frac{C_{0}^{2}}{\frac{2}{t^{p-2}}}, C_{1}^{2} \frac{\left\|u_{0, n}\right\|_{L^{1}(\Omega)}^{\frac{2 p}{N(p-p)}}}{t^{\frac{2 N}{N(p-2)+p}}}\right\}|\Omega| \\
& \quad+\frac{1}{\alpha p^{\prime}}\left(\max \left\{1, c_{P}\right\}\right)^{p^{\prime}}\left[\left\|f_{0}\right\|_{L^{p^{\prime}}\left(\Omega_{T}\right)}+\left\|f_{1}\right\|_{L^{p^{\prime}}\left(\Omega_{T}\right)}\right]^{p^{\prime}}, \quad \text { for every } 0<t \leq T,
\end{aligned}
$$

from which the assert (2.31) follows. To conclude the proof it remains to show that $u$ belongs also to $C\left((0, T) ; L^{2}(\Omega)\right) \cap C\left([0, T] ; L^{1}(\Omega)\right)$. The regularity $C\left((0, T) ; L^{2}(\Omega)\right)$ follows by the regularity $L_{\text {loc }}^{p}\left(0, T ; W_{0}^{1, p}(\Omega)\right)$ proved above together with the structure assumption (1.4). Finally, the regularity $C\left([0, T] ; L^{1}(\Omega)\right)$ can be proved proceeding exactly as in the proof of Theorem 1.7 (first step) in [21] and hence we omit it. 
Remark 4.2 We point out that the previous proof shows that under the assumptions of Theorem 2.5 every solution u constructed by approximation (i.e., as the limit of regular solutions $u_{n}$ of (4.33)) immediately increases its regularity, since it belongs to $C\left([0, T] ; L^{1}(\Omega)\right) \cap$ $C\left((0, T) ; L^{2}(\Omega)\right) \cap L_{l o c}^{p}\left(0, T ; W_{0}^{1, p}(\Omega)\right)$. Moreover, it also satisfies all the estimates stated in Theorem 2.5 .

\subsection{Proof of Theorem 2.6}

It is sufficient to prove estimate (2.34), since the uniqueness result immediately follows applying such an estimate to (eventually different) solutions belonging to $C\left([0, T] ; L^{1}(\Omega)\right) \cap$ $C\left((0, T) ; L^{2}(\Omega)\right) \cap L_{l o c}^{p}\left(0, T ; W_{0}^{1, p}(\Omega)\right)$ and satisfying the same initial datum $u_{0}$. Hence, let $u$ and $v$ as in the statement of the theorem. Now the proof of (2.34) follows proceeding exactly as in the proof of estimate (4.12) (simply replacing $u_{n}$ with $u$ and $v_{n}$ with $v$ in the proof above) since the regularity on $u$ and $v$ is sufficient to repeat exactly the same calculation done before.

\subsection{Proof of Theorem 2.7}

Let $u$ be a solution $u$ of (1.1) obtained as the a.e. limit in $\Omega_{T}$ of the solutions $u_{n} \in L^{\infty}\left(\Omega_{T}\right) \cap$ $C\left([0, T] ; L^{2}(\Omega)\right) \cap L^{p}\left(0, T ; W_{0}^{1, p}(\Omega)\right)$ of the following approximating problems

$$
\begin{cases}\left(u_{n}\right)_{t}-\operatorname{div}\left(a\left(x, t, \nabla u_{n}\right)\right)=f_{n}(x, t) & \text { in } \Omega_{T}, \\ u_{n}=0 & \text { on } \Gamma, \\ u_{n}(x, 0)=u_{0, n}(x) & \text { on } \Omega,\end{cases}
$$

where the data satisfy

$$
\begin{aligned}
& u_{0, n}(x) \in L^{\infty}(\Omega), \quad u_{0, n}(x) \rightarrow u_{0} \quad \text { in the weak-* topology of measures, } \\
& f_{n}(x, t) \in L^{\infty}\left(\Omega_{T}\right), \quad f_{n}(x, t) \rightarrow f \quad \text { in the weak- * topology of measures, }
\end{aligned}
$$

(see [7]). We recall that also in the case of measure data it results

$$
u \in L^{q}\left(0, T ; W_{0}^{1, q}(\Omega)\right) \quad \text { for every } \quad 1 \leq q<\frac{p(N+1)-N}{N+1}
$$

(see again [7]). Analogously, let $v \in L^{q}\left(0, T ; W_{0}^{1, q}(\Omega)\right.$ ) be a solution of (2.3) obtained as the a.e. limit in $\Omega_{T}$ of the sequence $v_{n} \in L^{\infty}\left(\Omega_{T}\right) \cap C\left([0, T] ; L^{2}(\Omega)\right) \cap L^{p}\left(0, T ; W_{0}^{1, p}(\Omega)\right)$ solutions of the following approximating problems

$$
\begin{cases}\left(v_{n}\right)_{t}-\operatorname{div}\left(a\left(x, t, \nabla v_{n}\right)\right)=f_{n}(x, t) & \text { in } \Omega_{T}, \\ v_{n}=0 & \text { on } \Gamma, \\ v_{n}(x, 0)=v_{0, n}(x) & \text { on } \Omega,\end{cases}
$$

where $f_{n}$ is the same approximating sequence defined in (4.48) and $v_{0, n}(x)$ satisfies

$$
v_{0, n}(x) \in L^{\infty}(\Omega) \quad v_{0, n}(x) \rightarrow v_{0} \quad \text { in the weak-* topology of measures. }
$$

We also choose the approximating sequence $u_{0, n}(x)$ verifying

$$
\left\|v_{0, n}(x)-u_{0, n}(x)\right\|_{L^{1}(\Omega)} \leq\left\|v_{0}-u_{0}\right\|_{M(\Omega)} .
$$


Choosing $G_{k}\left(u_{n}-v_{n}\right)(k>0)$ as test function in (4.46) and in (4.49) and proceeding exactly as in the proof of Theorem 2.1 we deduce that estimate (4.10) if $p>2$ and (4.12) if $p \geq 2$ hold true. Indeed, if $p=2$, proceeding as in the proof of (4.10) but applying Theorem 3.3 (instead of Theorem 3.2) we deduce that also the following estimate holds

$$
\left\|u_{n}(t)-v_{n}(t)\right\|_{L^{\infty}(\Omega)} \leq C_{2} \frac{\left\|u_{0, n}-v_{0, n}\right\|_{L^{1}(\Omega)}}{t^{\frac{N}{2}} e^{\sigma t}}
$$

where $C_{2}$ is a positive constant depending only on $N$ and $\alpha$ and $\sigma$ is given by the following formula

$$
\sigma=\frac{\alpha c_{s} \kappa}{2|\Omega|^{\frac{2}{N}}}, \quad \kappa \quad \text { arbitrarily fixed in }\left(0, \frac{1}{2}\right) .
$$

Hence, by the previous estimates and (4.51) it follows that for almost every $t \in(0, T)$

$$
\left\|u_{n}(t)-v_{n}(t)\right\|_{L^{\infty}(\Omega)} \leq \min \left\{\frac{C_{0}}{t^{\frac{1}{p-2}}}, C_{1} \frac{\left\|u_{0}-v_{0}\right\|_{M(\Omega)}^{\frac{p}{N(p-2)+p}}}{t^{\frac{N}{N(p-2)+p}}}\right\} \quad \text { if } p>2,
$$

and

$$
\left\|u_{n}(t)-v_{n}(t)\right\|_{L^{\infty}(\Omega)} \leq \min \left\{C_{2} \frac{\left\|u_{0}-v_{0}\right\|_{M(\Omega)}}{t^{\frac{N}{2}} e^{\sigma t}}, C_{1} \frac{\left\|u_{0}-v_{0}\right\|_{M(\Omega)}}{t^{\frac{N}{2}}}\right\} \quad \text { if } p=2,
$$

which imply the claims.

Remark 4.3 We notice that the proof above shows that if $u$ and $v$ are two solutions of, respectively (1.1) and (2.3), obtained as the limit (a.e. in $\Omega_{T}$ ) of solutions of the approximating problems (respectively) (4.46) and (4.49) with the approximating data $u_{0, n}, v_{0, n}$ and $f_{n}$ satisfying (4.47), (4.50) and (4.48), then if $p>2$ it results

$$
\|u(t)-v(t)\|_{L^{\infty}(\Omega)} \leq \frac{C_{0}}{t^{\frac{1}{p-2}}} \quad \text { for almost every } t \in(0, T) .
$$

Moreover, if we also assume (4.51), then also estimate (2.38) holds true if $p>2$ together with (2.39) if $p=2$.

\subsection{Proof of Theorem 2.8}

We observe that in the case of data in $L^{1}$, the construction of a global solution done in the proof of Theorem 2.2 above make use of the regularity $C\left([0, T] ; L^{1}(\Omega)\right)$ of the solution, which now fails having only measure data. Hence, it is necessary to proceed in a different way. The proof proceeds by steps.

Step 1 The goal of this step is the construction of a global solution $u$ of (1.1). To this aim, let us consider the following parabolic problems

$$
\begin{cases}\left(u_{n}\right)_{t}-\operatorname{div}\left(a\left(x, t, \nabla u_{n}\right)\right)=f_{n}(x, t) & \text { in } \Omega \times(0,+\infty) \\ u_{n}=0 & \text { on } \partial \Omega \times(0,+\infty), \\ u_{n}(x, 0)=u_{0, n}(x) & \text { on } \Omega\end{cases}
$$

where $u_{0, n}$ satisfies (4.47) and $f_{n}(x, t)$ belong to $L_{l o c}^{\infty}\left([0,+\infty) ; L^{\infty}(\Omega)\right)$ and satisfy for every $T>0: \quad f_{n}(x, t) \rightarrow f \quad$ in the weak- $*$ topology of measures on $\Omega_{T}$. 
We recall that for every fixed $n \in \mathbf{N}$, there exists a unique global solution $u_{n}$ of (4.55) in $L_{l o c}^{\infty}\left([0,+\infty) ; L^{\infty}(\Omega)\right) \cap C_{l o c}\left([0,+\infty] ; L^{2}(\Omega)\right) \cap L_{l o c}^{p}\left([0,+\infty) ; W_{0}^{1, p}(\Omega)\right)$. Moreover, for every arbitrarily fixed $T>0$, there exists a subsequence of the sequence $u_{n}$ converging a.e. in $\Omega_{T}$ (indeed a stronger convergence holds) to a solution of our problem (1.1) in $\Omega_{T}$ (see [7]). Hence, let $T_{1}>0$ arbitrarily fixed. As said above, there exists a subsequence of $u_{n}$, that we denote $u_{n}^{(1)}$, such that

$$
u_{n}^{(1)} \rightarrow u_{1} \text { a.e. in } \Omega_{T_{1}}
$$

where $u_{1}$ is a solution of our problem (1.1) in $\Omega_{T_{1}}$. We recall that every term of the sequence $u_{n}^{(1)}$ is a global solution, and thus it is also a solution in $\Omega_{2 T_{1}}$. Hence, again by the results in [7], there exists a subsequence of $u_{n}^{(1)}$, that we denote $u_{n}^{(2)}$, such that

$$
u_{n}^{(2)} \rightarrow u_{2} \text { a.e. in } \Omega_{2 T_{1}}
$$

where $u_{2}$ is a solution of our problem (1.1) in $\Omega_{2 T_{1}}$.

Iterating this procedure, we define a function $u$ in $\Omega \times(0,+\infty)$ in the following way

$$
\text { for every } T>0: u(x, t)=u_{m}(x, t) \text { a.e. in } \Omega_{T},
$$

where $m \in \mathbf{N}$ is such that $m T_{0}>T$. We notice that the definition of $u$ is well posed since (by construction) if $h \in \mathbf{N}$ is such that $h T_{0}>T$ then it results

$$
u_{m}(x, t)=u_{h}(x, t) \text { a.e. in } \Omega_{T} .
$$

We point out that for every arbitrarily fixed $T>0 u$ solves (1.1) and hence is a global solution.

Step 2 We conclude here the proof of the theorem. Let $v$ be the global solution constructed exactly as in the previous step but only changing the approximating sequence $u_{0, n}$ with a sequence $v_{0, n}$ satisfying (4.50) and (4.51) (hence we do not change the approximating sequence $f_{n}$ of $\mathrm{f}$ ). Let now $T>0$ arbitrarily fixed. Proceeding exactly as in the proof of Theorem 2.7 we deduce that estimate (2.38) if $p>2$ and (2.39) if $p=2$ hold true. Hence the assertion follows by the arbitrariness of $T$.

\subsection{Proof of Corollary 2.3}

As just recalled above, under the assumptions of Corollary 2.3 there exists a solution $\mathrm{w}$ of the stationary problem (2.14). Moreover, w can be constructed as the a.e. limit in $\Omega$ (indeed further convergences are true) of the solutions $w_{n}$ of (2.15) with $f_{n}$ satisfying

$$
f_{n} \in L^{\infty}(\Omega), \quad f_{n}(x) \rightarrow f \quad \text { in the weak- * topology of measures. }
$$

Let now $u$ be the global solution of (1.1) constructed as in the proof of Theorem 2.8 but using the same approximation $f_{n}$ of $\mathrm{f}$ done above to construct $\mathrm{w}$ (this is possible being $\mathrm{f}$ independent of the variable $\mathrm{t}$ ). It is not restrictive to assume also that the approximation sequence $u_{0, n}$ in (4.55) satisfies

$$
\left\|u_{0, n}-w_{n}\right\|_{L^{1}(\Omega)} \leq\left\|u_{0}-w\right\|_{M(\Omega)} .
$$

Now the proof proceeds similarly at all to that of Corollary 2.1 and hence we omit it. 


\subsection{Proof of Theorem 2.9}

The proof is similar at all to that of Theorem 2.3. Let $\mathrm{v}$ as in the statement of the theorem a solution of (2.3) constructed as the limit (a.e. in $\Omega_{T}$ ) of $v_{n}$ solutions in $L^{\infty}\left(\Omega_{T}\right) \cap C\left([0, T] ; L^{2}(\Omega)\right) \cap L^{p}\left(0, T ; W_{0}^{1, p}(\Omega)\right)$ of (2.46) with $f_{n}$ as in (2.47). By Theorem 2.7 and its proof we deduce that there exists a solution $u$ of (1.1) satisfying (2.38) if $p>2$ and (2.39) if $p=2$. Consequently, it results for almost every $(x, t) \in \Omega_{T}$

$$
|u(x, t)| \leq|v(x, t)|+\min \left\{\frac{C_{0}}{t^{\frac{1}{p-2}}}, C_{1} \frac{\left\|u_{0}\right\|_{M(\Omega)}^{\frac{p}{N(p-2)+p}}}{t^{\frac{N}{N(p-2)+p}}}\right\} \quad \text { if } p>2
$$

and

$$
|u(x, t)| \leq|v(x, t)|+\min \left\{C_{2} \frac{\left\|u_{0}\right\|_{M(\Omega)}}{t^{\frac{N}{2}} e^{\sigma t}}, C_{1} \frac{\left\|u_{0}\right\|_{M(\Omega)}}{t^{\frac{N}{2}}}\right\} \quad \text { if } p=2
$$

Now all the assertions are immediate consequences of the previous estimates.

\subsection{Proof of Theorem 2.10}

Let us choose $v_{0}=0$ and $v=u^{0}$, where $u^{0}$ is, as above, the solution of (1.14) obtained as limit of the approximating solutions $u_{n}^{0}$ of (2.17). We recall that $u^{0}$ is bounded and satisfies the $L^{\infty}$. estimate (4.27). Hence, by Theorem 2.9 there exists a solution $u$ (obtained by approximation) satisfying

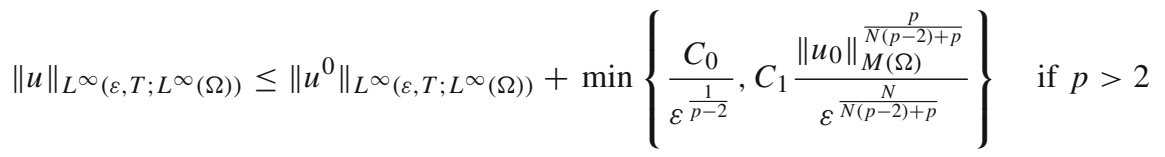

and

$\|u\|_{L^{\infty}\left(\varepsilon, T ; L^{\infty}(\Omega)\right)} \leq\left\|u^{0}\right\|_{L^{\infty}\left(\varepsilon, T ; L^{\infty}(\Omega)\right)}+\min \left\{C_{2} \frac{\left\|u_{0}\right\|_{M(\Omega)}}{\varepsilon^{\frac{N}{2}} e^{\sigma \varepsilon}}, C_{1} \frac{\left\|u_{0}\right\|_{M(\Omega)}}{\varepsilon^{\frac{N}{2}}}\right\} \quad$ if $p=2$

Now the proof of the theorem proceeds exactly as that of Theorem 2.4 and hence we omit it.

\subsection{Proof of Theorem 2.11}

The proof follows closely the outline of the proof of Theorem 2.5 and hence we only describe the changes. Let $u^{0}$ be the unique solution of (1.14) belonging to $C\left([0, T] ; L^{2}(\Omega)\right) \cap$ $L^{p}\left(0, T ; W_{0}^{1, p}(\Omega)\right)$. Since the datum $\mathrm{f}$ is sufficiently regular, we can construct $u$ by approximating only the initial datum $u_{0}$. In detail, let $u$ be the almost everywhere limit in $\Omega_{T}$ of the solution $u_{n}$ of (4.33) where we choose the sequence $u_{0, n}$ satisfying (4.47) and the following bound

$$
\left\|u_{0, n}\right\|_{L^{1}(\Omega)} \leq\left\|u_{0}\right\|_{M(\Omega)} .
$$

We point out that the regularity of $u_{n}$ and $u^{0}$ is enough to conclude (choosing $G_{k}\left(u_{n}-u^{0}\right.$ ) as test functions in (4.33) and in (1.14) and proceeding exactly as in the proof Theorem 2.7) that estimates (4.53) and (4.54) hold true with $v_{n}$ replaced by $u^{0}$, i.e., it results 


$$
\left\|u_{n}(t)-u^{0}(t)\right\|_{L^{\infty}(\Omega)} \leq \min \left\{\frac{C_{0}}{t^{\frac{1}{p-2}}}, C_{1} \frac{\left\|u_{0}\right\|_{M(\Omega)}^{\frac{p}{N(p-2)+p}}}{t^{\frac{N}{N(p-2)+p}}}\right\} \quad \text { if } p>2,
$$

and

$$
\left\|u_{n}(t)-u^{0}(t)\right\|_{L^{\infty}(\Omega)} \leq \min \left\{C_{2} \frac{\left\|u_{0}\right\|_{M(\Omega)}}{t^{\frac{N}{2}} e^{\sigma t}}, C_{1} \frac{\left\|u_{0}\right\|_{M(\Omega)}}{t^{\frac{N}{2}}}\right\} \quad \text { if } p=2,
$$

with $C_{0}$ and $C_{1}$ as in (2.5) and $C_{2}$ and $\sigma$ as in (2.39). Hence, by the previous estimates we deduce (proceeding as in the proof of Theorem 2.9) that for every $\varepsilon \in(0, T)$ the following estimates hold

$$
\begin{aligned}
& \left\|u_{n}\right\|_{L^{\infty}\left(\varepsilon, T ; L^{2}(\Omega)\right)} \leq\left\|u^{0}\right\|_{L^{\infty}\left(\varepsilon, T ; L^{2}(\Omega)\right)}+\min \left\{\frac{C_{0}}{t^{\frac{1}{p-2}}}, C_{1} \frac{\left\|u_{0}\right\|_{M(\Omega)}^{\frac{p}{N(p-2)+p}}}{t^{\frac{N}{N(p-2)+p}}}\right\}|\Omega|^{\frac{1}{2}} \quad \text { if } p>2, \\
& \left\|u_{n}\right\|_{L^{\infty}\left(\varepsilon, T ; L^{2}(\Omega)\right)} \leq\left\|u^{0}\right\|_{L^{\infty}\left(\varepsilon, T ; L^{2}(\Omega)\right)}+\min \left\{C_{2} \frac{\left\|u_{0}\right\|_{M(\Omega)}}{t^{\frac{N}{2}} e^{\sigma t}}, C_{1} \frac{\left\|u_{0}\right\|_{M(\Omega)}}{t^{\frac{N}{2}}}\right\}|\Omega|^{\frac{1}{2}} \quad \text { if } p=2, \\
& \|u\|_{L^{\infty}\left(\varepsilon, T ; L^{2}(\Omega)\right)} \leq\left\|u^{0}\right\|_{L^{\infty}\left(\varepsilon, T ; L^{2}(\Omega)\right)}+\min \left\{\frac{C_{0}}{t^{\frac{1}{p-2}}}, C_{1} \frac{\left\|u_{0}\right\|_{M(\Omega)}^{\frac{p}{N(p-2)+p}}}{t^{\frac{N}{N(p-2)+p}}}\right\}|\Omega|^{\frac{1}{2}} \quad \text { if } p>2,
\end{aligned}
$$

and

$$
\|u\|_{L^{\infty}\left(\varepsilon, T ; L^{2}(\Omega)\right)} \leq\left\|u^{0}\right\|_{L^{\infty}\left(\varepsilon, T ; L^{2}(\Omega)\right)}+\min \left\{C_{2} \frac{\left\|u_{0}\right\|_{M(\Omega)}}{t^{\frac{N}{2}} e^{\sigma t}}, C_{1} \frac{\left\|u_{0}\right\|_{M(\Omega)}}{t^{\frac{N}{2}}}\right\}|\Omega|^{\frac{1}{2}} \quad \text { if } p=2 .
$$

Hence, estimate (2.57) follows by the previous estimates recalling the $L^{\infty}\left(\varepsilon, T ; L^{2}(\Omega)\right)$ estimate (4.39) of $u^{0}$.

Now the remaining estimate (2.58) on the gradient of $u$ follows proceeding as in the proof of the bound (2.31) of Theorem 2.5, i.e., combining the $L^{\infty}\left(\varepsilon, T ; L^{2}(\Omega)\right)$-estimates above with (4.41) and (4.43).

\section{References}

1. Andreu, F., Mazon, J.M., Segura De Leon, S., Toledo, J.: Existence and uniqueness for a degenerate parabolic equation with $L^{1}(\Omega)$ data. Trans. Am. Math. Soc. 351, 285-306 (1999)

2. Aronson, D.G., Serrin, J.: Local behavior of solutions of quasilinear parabolic equations. Arch. Ration. Mech. Anal. 25, 81-122 (1967)

3. Blanchard, D., Murat, F.: Renormalized solutions of nonlinear parabolic problems with $L^{1}$ data, Existence and uniqueness. Proc. R. Soc. Edimburgh Sect. A 127, 1137-1152 (1997)

4. Blanchard, D., Murat, F., Redwane, H.: Existence and uniqueness of a renormalized solution for a fairly general class of nonlinear parabolic problems. J. Differ. Equ. 177, 331-374 (2001)

5. Blanchard, D., Redwane, H.: Renormalized solutions for a class of nonlinear evolution problems. J. Math. Pure Appl. 77, 117-151 (1998)

6. Boccardo, L., Dall'Aglio, A., Gallouet, T., Orsina, L.: Existence and regularity results for nonlinear parabolic equations. Adv. Math. Sci. Appl. 9, 1017-1031 (1999)

7. Boccardo, L., Gallouet, T.: Nonlinear elliptic and parabolic equations involving measure data. J. Funct. Anal. 87, 149-169 (1989)

8. Boccardo, L., Porzio, M.M., Primo, A.: Summability and existence results for nonlinear parabolic equations. Nonlinear Anal TMA 71, 978-990 (2009)

9. Bonforte, M., Grillo, G.: Super and ultracontractive bounds for doubly nonlinear evolution equations. Rev. Mat. Iberoamericana 22(1), 11-129 (2006) 
10. Cipriani, F., Grillo, G.: Uniform bounds for solutions to quasilinear parabolic equations. J. Differ. Equ. 177, 209-234 (2001)

11. Dall'Aglio, A.: Approximated solutions of equations with $L^{1}$ data. Application to the $H$-convergence of quasi-linear parabolic equations. Ann. Mat. Pura Appl. 170, 207-240 (1996)

12. Di Benedetto, E.: Degenerate Parabolic Equations. Springer, New York (1993)

13. Di Benedetto, E., Herrero, M.A.: On the Cauchy problem and initial traces for a degenerate parabolic equation. Trans. AMS 314, 187-224 (1989)

14. Herrero, M.A., Vazquez, J.L.: Asymptotic behaviour of the solutions of a strongly nonlinear parabolic problem. Ann. Fac. Sci. Toulose Math. (5) 3(2), 113-127 (1981)

15. Kalashnikov, A.S.: Cauchy's problem in classes of increasing functions for certain quasi-linear degenerate parabolic equations of the second order. Differ. Uravneniya 9(4), 682-691 (1973)

16. Ladyženskaja, O., Solonnikov, V.A., Ural'ceva, N.N.: Linear and Quasilinear Equations of Parabolic Type. Translations of the American Mathematical Society. American Mathematical Society, Providence (1968)

17. Porzio, M.M.: $L_{l o c}^{\infty}$-estimates for degenerate and singular parabolic equations. Nonlinear Anal Theory Methods Appl 17(11), 1093-1107 (1991)

18. Porzio, M.M.: Existence of solutions for some "noncoercive" parabolic equations. Discrete Continuous Dyn Syst 5(3), 553-568 (1999)

19. Porzio, M.M.: Local regularity results for some parabolic equations. Houston J. Math. 25(4), 769-792 (1999)

20. Porzio, M.M.: On decay estimates. J. Evol. Equ. 9(3), 561-591 (2009)

21. Porzio, M.M.: Existence, uniqueness and behavior of solutions for a class of nonlinear parabolic problems. Nonlinear Anal. TMA 74, 5359-5382 (2011)

22. Porzio, M.M.: On uniform and decay estimates for unbounded solutions of partial differential equations. J. Differ. Equ. 259, 6960-7011 (2015)

23. Porzio, M.M.: Regularity and time behavior of the solutions of linear and quasilinear parabolic equations. Adv. Differ. Equ. 23(5-6), 329-372 (2018)

24. Porzio, M.M.: A new approach to decay estimates. Application to a nonlinear and degenerate parabolic PDE. Rend. Lincei Mat. Appl. 29, 635-659 (2018)

25. Porzio, M.M.: Quasilinear parabolic and elliptic equations with singular potentials. In: Wood, D.R., de Gier, J., Praeger, C.E., Tao, T. (eds.) 2017 MATRIX Annals. MATRIX Book Series, vol 2. Springer, Cham (2019)

26. Porzio, M.M.: Regularity and time behavior of the solutions to weak monotone parabolic equations (preprint)

27. Porzio, M.M., Pozio, M.A.: Parabolic equations with non-linear, degenerate and space-time dependent operators. J. Evol. Equ. 8, 31-70 (2008)

28. Prignet, A.: Existence and uniqueness of "entropy" solutions of parabolic problems with $L^{1}$ data. Nonlinear Anal. TMA 28(12), 1943-1954 (1997)

29. Vazquez, J.L.: Smoothing and Decay Estimates for Nonlinear Diffusion Equations. Oxford University Press, Oxford (2006)

30. Veron, L.: Effects regularisants des semi-groupes non linéaires dans des espaces de Banach. Ann. Fac. Sci. Toulose Math. (5) 1(2), 171-200 (1979)

Publisher's Note Springer Nature remains neutral with regard to jurisdictional claims in published maps and institutional affiliations. 